\title{
Relic density of wino-like dark matter in the MSSM
}

\section{Beneke, ${ }^{a}$ A. Bharucha, ${ }^{b}$ F. Dighera, ${ }^{a}$ C. Hellmann, ${ }^{a}$ A. Hryczuk,,${ }^{a, c, d}$ S. Recksiegel ${ }^{a}$ and P. Ruiz-Femenía ${ }^{a}$}

${ }^{a}$ Physik Department T31, Technische Universität München, James-Franck-Straße, D-85748 Garching, Germany

${ }^{b}$ CPT, Aix-Marseille Université, Université de Toulon, CNRS, Case 90\%, F-13288 Marseille, France

${ }^{c}$ National Centre for Nuclear Research, Hoża 69, 00-681, Warsaw, Poland

${ }^{d}$ Department of Physics, University of Oslo, Box 1048, NO-0371 Oslo, Norway

E-mail: aoife.bharucha@cpt.univ-mrs.fr, francesco.dighera@tum.de, a.j.hryczuk@fys.uio.no, stefan.recksiegel@tum.de, pedro.ruiz-femenia@tum.de

ABSTRACT: The relic density of TeV-scale wino-like neutralino dark matter in the MSSM is subject to potentially large corrections as a result of the Sommerfeld effect. A recently developed framework enables us to calculate the Sommerfeld-enhanced relic density in general MSSM scenarios, properly treating mixed states and multiple co-annihilating channels as well as including off-diagonal contributions. Using this framework, including on-shell oneloop mass splittings and running couplings and taking into account the latest experimental constraints, we perform a thorough study of the regions of parameter space surrounding the well known pure-wino scenario: namely the effect of sfermion masses being non-decoupled and of allowing non-negligible Higgsino or bino components in the lightest neutralino. We further perform an investigation into the effect of thermal corrections and show that these can safely be neglected. The results reveal a number of phenomenologically interesting but so far unexplored regions where the Sommerfeld effect is sizeable. We find, in particular, that the relic density can agree with experiment for dominantly wino neutralino dark matter with masses ranging from 1.7 to beyond $4 \mathrm{TeV}$. In light of these results the bounds from Indirect Detection on wino-like dark matter should be revisited.

KEYwORDS: Supersymmetry Phenomenology

ARXIV EPRINT: 1601.04718 


\section{Contents}

1 Introduction $\quad 2$

2 MSSM parameters, constraints and implementation of the Sommerfeld effect

2.1 MSSM definition and parameter ranges

2.2 Constraints

2.2.1 Collider and flavour constraints

2.2.2 Theoretical constraints

2.2.3 Cosmological and direct DM detection constraints

$\begin{array}{lll}2.3 & \text { One-loop mass splittings } & 10\end{array}$

2.4 Running couplings 11

2.5 Annihilation matrix implementation 12

$\begin{array}{lll}2.6 & \text { Sommerfeld-corrected cross section } & 16\end{array}$

3 Analysis 18

$\begin{array}{lll}3.1 & \text { Impact of sfermions } & 18\end{array}$

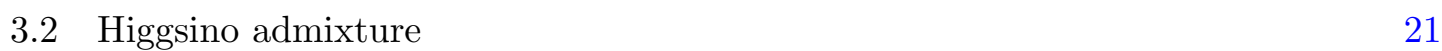

3.3 Effect of the heavy Higgs bosons 24

$\begin{array}{lll}3.4 & \text { Bino admixture } & 26\end{array}$

$\begin{array}{lll}3.5 & \text { Residual dependence on other parameters } & 29\end{array}$

$\begin{array}{lll}4 & \text { Summary } & 34\end{array}$

A Thermal effects $\quad 35$

A.1 Higgs vacuum expectation value 36

$\begin{array}{ll}\text { A.1.1 Gauge boson masses } & 36\end{array}$

$\begin{array}{lll}\text { A.1.2 Lightest neutralino-chargino mass difference } & 37\end{array}$

$\begin{array}{lll}\text { A.2 Thermal self-energies } & 38\end{array}$

$\begin{array}{lll}\text { A.2.1 Electroweak gauge bosons } & 38\end{array}$

$\begin{array}{lll}\text { A.2.2 Neutralino-chargino mass difference } & 39\end{array}$

A.3 Effect on the Sommerfeld enhancement and relic density 40

A.3.1 Yukawa potential 41

A.3.2 Neutralino-chargino mass splitting 41

$\begin{array}{lll}\text { A.3.3 Summary } & 42\end{array}$ 


\section{Introduction}

The so-called "WIMP miracle" is the observation that a thermally produced, stable, massive particle $\chi$ with electroweak interactions (WIMP) naturally accounts for the observed dark matter relic density, if its mass is of order of the electroweak or $\mathrm{TeV}$ scale. Indeed, on adding a fermionic SU(2) triplet to the Standard Model (SM), its tree-level pair annihilation into electroweak gauge bosons yields $\Omega_{\mathrm{cdm}} h^{2}=0.1188$ for $m_{\chi} \approx 2.2 \mathrm{TeV}$. Such models provide attractive dark matter (DM) candidates due to their minimal particle content [1], but further model building is required to explain why the mass of the $\chi$ particle should be close to the electroweak scale. The minimal supersymmetric standard model (MSSM) is a prime example of a model where the DM particle mass is tied to the electroweak scale by the desire to temper the quantum corrections to the Higgs. The underlying symmetry principle then leads to a proliferation of particles and interactions, allowing for different successful DM candidates. Within this context thermal scenarios with DM masses below $1 \mathrm{TeV}$ require additional mechanisms or accidental degeneracies, e.g. resonant annihilation or coannihilation, in order to avoid overproduction in the early Universe. These are also becoming somewhat constrained by LHC and dark matter searches, for recent analyses see refs. [25]. Scenarios with heavier dark matter interpolate to minimal models, since the supersymmetric particles form approximate electroweak multiplets (except for degeneracies). In particular, when the lightest supersymmetric particle ("wino") is the partner of the electroweak gauge bosons, the model is similar to the minimal triplet model, but modifications arise due to the mixing with the Higgsino and bino states, as well as the interactions with sfermions. It is this "wino-like" region of the MSSM parameter space, which we focus on in this paper.

The wino-like region deserves special attention, since the DM relic density cannot be calculated reliably from the tree-level annihilation cross section. Loop effects from electroweak gauge boson exchange are large in non-relativistic scattering before the annihilation of TeV-scale dark matter, and lead to the electroweak Sommerfeld effect [6, 7], which is particularly strong in the wino-like region. This has been studied extensively in the purewino limit [6-10], which corresponds to the minimal triplet model. To be specific, in the analysis below we find that the observed relic density is attained at significantly larger mass $m_{\chi}=2.88 \mathrm{TeV}$ when the Sommerfeld effect is accounted for, instead of $2.22 \mathrm{TeV}$ at tree level, when $M_{1}=3 M_{2}, \mu=2 M_{2}$ and the common sfermion mass $M_{\mathrm{sf}}=20 \mathrm{TeV}$, which corresponds effectively to the pure-wino limit. The Sommerfeld effect also displays a resonance at $2.33 \mathrm{TeV}$, where the relic density is reduced by a factor 3.9 relative to the computation based on the tree-level cross section. This highlights the importance of including the Sommerfeld effect in full MSSM calculations of the relic density in the wino-like region.

Away from the pure-wino limit, the lightest neutralino is a mixture of wino, Higgsino and bino eigenstates and interacts accordingly, which makes the computation of the Sommerfeld effect much more involved. This problem was first approached in refs. [9, 11], however a framework that deals systematically with mixed states, multiple co-annihilating states and the corresponding off-diagonal reactions was only developed in refs. [12-14], which allowed the computation of the relic density including the Sommerfeld effect with a relative accuracy similar to state-of-the-art computations employing Born cross sections. 
This was studied in a number of models that interpolate from a pure-wino to a pureHiggsino DM particle [15], but a detailed investigation of the MSSM parameter space was left for the future.

We report on this investigation in the present work, focusing on the wino-like region of the full MSSM. We note that in this region the Sommerfeld effect is not a small correction and should be included in any reliable relic density computation and in particular when the relic density is correlated with other observational constraints. The most important is from indirect dark matter searches. For instance, the thermal pure-wino scenario is often said to be excluded (barring some astrophysical uncertainties, see ref. [16]) by the non-observation of a photon line signal from the Galactic Centre [16-18]. Other search channels, especially the cosmic ray antiprotons and the diffuse gamma rays from dwarf spheroidal galaxies, also start to give competitive limits [16, 19-21].

This conclusion need not hold in the full MSSM, when the mixed nature of wino-like dark matter is taken into account. The framework adopted here follows refs. [12-14], with several improvements applied relative to ref. [15]. We now include the running of the electroweak couplings from the electroweak to the dark matter scale, and use the exact one-loop neutralino and chargino on-shell masses to compute the mass splitting, which is important in the resonance region. We justify neglecting thermal effects due to the fact that the freeze-out happens at temperatures close to the electroweak scale. On the practical side, a considerable speed-up of the numerical evaluation has been achieved, which now allows a systematic investigation of the relevant MSSM parameter space in the wino-like region.

The outline of the paper is as follows: in section 2 we define the ranges of the parameters of the phenomenological MSSM, and discuss the theoretical and observational constraints we apply to select viable models. We further briefly summarize the computation of the Sommerfeld correction, the implementation of mass splittings and the running of the electroweak coupling. The set-up is rather general, but the present version does not include sfermion-neutralino/chargino potentials and hence excludes models with sfermion co-annihilation, as well as s-channel resonant annihilation, in which case the annihilation process is not short-distance. Section 3 contains our main results. Here we show and discuss, in order, the dependence of the relic density and the relative importance of the Sommerfeld effect on the sfermion masses $M_{\mathrm{sf}}$ (all assumed degenerate for simplicity), on the heavy MSSM Higgs bosons, further on the Higgsino admixture via the difference $\mu-M_{2}$ of the Higgsino and wino mass parameters of the MSSM, and similarly on the bino admixture. We shall see that away from the pure-wino limit, the observed relic density is obtained for a wide range of wino-like dark matter particle masses and we quantify and explain the parameter dependence. We further study the dependence on other MSSM parameters, which generally turns out to be minor, except in the vicinity of the Sommerfeld resonance. We summarize in section 4 .

The investigation of thermal effects is contained in appendix A. We consider the temperature dependence of the electroweak gauge boson masses, which in turn affects the range of the electroweak Yukawa potential, and of the neutralino-chargino mass difference. The dependence arises from the temperature-dependent Higgs vacuum expectation value and the one-loop self-energies. Despite the fact that freeze-out may begin in the symmetric 
phase of the electroweak interactions, where the Higgs field has no expectation value and the thermal effects are large, we find that the impact on the relic density is negligible within other uncertainties. We explain why previous work $[8,9]$ overemphasised the effect.

\section{MSSM parameters, constraints and implementation of the Sommerfeld effect}

\subsection{MSSM definition and parameter ranges}

We are interested in exploring the parameter space of the CP-conserving, minimal flavour violating MSSM defined at the electroweak scale. Within this space we focus primarily on the calculation of the relic abundance of neutralino dark matter in the close-to-wino region, which only depends strongly on a subset of parameters. Clearly a central role is played by those parameters describing the chargino and neutralino sector: the bino mass $M_{1}$, the wino mass $M_{2}$ and the Higgsino parameter $\mu .^{1}$ The tree level mass matrix for the charginos is given by

$$
X=\left(\begin{array}{cc}
M_{2} & \sqrt{2} m_{W} s_{\beta} \\
\sqrt{2} m_{W} c_{\beta} & \mu
\end{array}\right),
$$

where $s_{\beta} / c_{\beta} \equiv \sin \beta / \cos \beta, \tan \beta$ being the ratio of the vevs of the two MSSM Higgs doublets, and $m_{W}$ is the mass of the $W$ boson. The mass matrix for the neutralinos is given by

$$
Y=\left(\begin{array}{cccc}
M_{1} & 0 & -m_{Z} c_{\beta} s_{W} & m_{Z} s_{\beta} s_{W} \\
0 & M_{2} & m_{Z} c_{\beta} c_{W} & -m_{Z} s_{\beta} c_{W} \\
-m_{Z} c_{\beta} s_{W} & m_{Z} c_{\beta} c_{W} & 0 & -\mu \\
m_{Z} s_{\beta} s_{W} & -m_{Z} s_{\beta} c_{W} & -\mu & 0
\end{array}\right) .
$$

where $s_{W} \equiv \sin \theta_{W}, c_{W} \equiv \cos \theta_{W}$ for the Weinberg angle $\theta_{W}$ and $m_{Z}$ is the mass of the $Z$ boson. On diagonalising the hermitian squares of these matrices one obtains the values of the masses of the charginos and neutralinos $m_{\tilde{\chi}_{i}^{+}}$and $m_{\tilde{\chi}_{j}^{0}}$ respectively, numbered $i=1,2, j=1, \ldots, 4$ in increasing order.

We concentrate on the region where the lightest supersymmetric particle (LSP) mass is at the $\mathrm{TeV}$ scale, as this is where the wino-like neutralino can provide the correct thermal relic density and the electroweak Sommerfeld effect is non-negligible. Here we assume that either the bino, Higgsino or both are much heavier than the wino. We can study the mixing angles of the wino with the bino or the Higgsinos and the resulting mass eigenstates by expanding in $m_{Z} / \mu$ etc. The mixing as well as the mass difference between the lightest chargino and neutralino can play an important role in determining the size of the Sommerfeld enhancement. In the region where the bino is decoupled, provided that $m_{W} \ll|\mu|-M_{2}$, the splitting $\delta m_{\tilde{\chi}_{1}^{+}} \equiv m_{\tilde{\chi}_{1}^{+}}-m_{\tilde{\chi}_{1}^{0}}$ is given by

$$
\delta m_{\tilde{\chi}_{1}^{+}} \simeq \frac{1}{2} \frac{m_{W}^{4} M_{2}\left(c_{\beta}^{2}-s_{\beta}^{2}\right)^{2}}{\left(\mu^{2}-M_{2}^{2}\right)^{2}} .
$$

\footnotetext{
${ }^{1}$ Note that we adopt a convention where the sign of $M_{2}$ is positive, but vary that of $M_{1}$ and $\mu$.
} 
If the difference $\delta \mu \equiv|\mu|-M_{2}$ is too small, the splitting is found to be

$$
\delta m_{\tilde{\chi}_{1}^{+}} \simeq \frac{m_{Z}^{2}}{8 M_{2}}\left(c_{W}^{2}\left(1 \mp s_{2 \beta}\right)\left(1-\frac{\delta \mu}{\sqrt{2}\left(s_{\beta} \pm c_{\beta}\right) m_{W}}\right)+2 s_{W}^{2}\left(1 \pm s_{2 \beta}\right) \frac{M_{2}}{M_{1}}\right)
$$

where the upper (lower) sign corresponds to positive (negative) $\mu$. We have kept the leading sub-leading correction for large $\left|M_{1}\right|$, but dropped terms of order $m_{Z}^{2} \delta \mu /\left(M_{2}\left|M_{1}\right|\right)$.

When the Higgsinos are decoupled, if $s_{2 \beta} m_{Z}^{2} \ll 2|\mu|\left|\delta M_{1}\right|$, where $\delta M_{1} \equiv M_{1}-M_{2}$, the mixing between the wino and bino depends on

$$
\theta_{b}=\frac{s_{2 \beta} s_{2 W} m_{Z}^{2}}{2 \mu \delta M_{1}}
$$

where $s_{2 W} \equiv \sin 2 \theta_{W}$. Note that for negative $M_{1},\left|\delta M_{1}\right|$ is not a small quantity, the mixing is suppressed and the tree-level splitting between the lightest chargino and neutralino is negligible; we do not discuss this case further here. Then, depending on whether $s_{2 \beta} m_{Z}^{2} \ll$ $2|\mu| \delta M_{1}$ or $s_{2 \beta} m_{Z}^{2} \gg 2|\mu| \delta M_{1}$,

$$
\begin{aligned}
& \delta m_{\tilde{\chi}_{1}^{+}} \simeq \theta_{b}^{2} \delta M_{1}\left(1+\frac{2 M_{2}}{s_{2 \beta} \mu}\right) \text { or } \\
& \delta m_{\tilde{\chi}_{1}^{+}} \simeq\left\{\begin{array}{l}
s_{W}^{2} \frac{m_{Z}^{2}}{\mu}\left(s_{2 \beta}+\frac{M_{2}}{\mu}\right)-s_{W}^{2} \delta M_{1}, \text { if } \mu>0 \text { or } \frac{s_{2 \beta}|\mu|}{M_{2}}<1 \\
c_{W}^{2} \frac{m_{Z}^{2}}{|\mu|}\left(s_{2 \beta}+\frac{M_{2}}{\mu}\right)-c_{W}^{2} \delta M_{1}, \text { otherwise }
\end{array}\right.
\end{aligned}
$$

respectively. In eq. (2.6), there is a clear decrease in $\delta m_{\tilde{\chi}_{1}^{+}}$as $\delta M_{1}$ increases. Apart from this, one finds that the mass splitting decreases (when $\mu$ is positive) as $\tan \beta$ increases, or as $\mu$ increases. Also, under the assumptions where eq. (2.6) and the first of eq. (2.7) hold, for the same value of $|\mu|$ the mass splitting is always smaller for $\mu<0$ than for $\mu>0$. The only remaining gaugino is the gluino, the mass of which is determined by the parameter $M_{3}$. The value of this parameter does not have much effect on our results, provided it is sufficiently heavy.

The sfermions can play a non-negligible role in the annihilation. The sfermion mass matrix is given by

$$
M_{\tilde{f}}=\left(\begin{array}{cc}
M_{\tilde{f}_{L}}^{2}+m_{f}^{2}+\tilde{m}_{Z}^{2}\left(I_{3}^{f}-Q_{f} s_{W}^{2}\right) & m_{f} X_{f}^{*} \\
m_{f} X_{f} & M_{\tilde{f}_{R}}^{2}+m_{f}^{2}+\widetilde{m}_{Z}^{2} Q_{f} s_{W}^{2}
\end{array}\right),
$$

for right- and left-handed sfermion mass parameters $M_{\tilde{f}_{L}}, M_{\tilde{f}_{R}}$. We make use of the abbreviation $\widetilde{m}_{Z}^{2} \equiv m_{Z}^{2} \cos 2 \beta$, and $X_{f}$ is defined in terms of the trilinear coupling $A_{f}$ via

$$
X_{f} \equiv A_{f}-\mu^{*}\{\cot \beta, \tan \beta\},
$$

where $\cot \beta$ applies for the up-type squarks, $f=u, c, t$, and $\tan \beta$ applies for the downtype sfermions, $f=d, s, b, e, \mu, \tau$ (we treat the neutrinos as being massless). Note that 
$m_{f}, Q_{f}$ and $I_{3}^{f}$ are the mass, charge and isospin projection of the fermion $f$ respectively. We significantly simplify the sfermion sector by adopting a common mass parameter $M_{\mathrm{sf}} \equiv M_{\tilde{f}_{L}}=M_{\tilde{f}_{R}}$. This simplification is justified in that it does not introduce any nontrivial modification of the $\mathrm{DM}$ properties, i.e. at the $\mathrm{TeV}$ scale close to the wino limit the dominant contribution to the annihilation cross section involves gauge interactions and gauge universality implies equal contributions from all flavours. Therefore, the effect of introducing more freedom in the sfermion masses can be estimated by a simple rescaling of the effect. The sfermion mass parameter is taken to be always larger by at least $25 \%$ than $M_{2}$, in order not to have sfermion co-annihilation processes. ${ }^{2}$

The MSSM Higgs sector consists of two doublets $\Phi_{1}$ and $\Phi_{2}$, which after electroweak symmetry breaking leads to three neutral particles, the light neutral and the heavy CPodd and CP-even Higgs bosons, as well as a heavy charged Higgs boson. Their masses and mixing can be completely described in terms of two parameters, $\tan \beta$ introduced earlier and the mass $M_{A}$ of the CP-odd neutral Higgs boson $A^{0}$, which defines the mass scale of the heavy doublet $\Phi_{2}$. The masses of the remaining particles and the mixing between them can be deduced from these two parameters, on requiring the minimisation of the Higgs potential. Any CP phases that could arise in the Higgs sector may be rotated away, and the Higgs sector is CP-conserving at tree level.

The ranges of the MSSM parameters are assumed to be as wide as possible within the experimentally and theoretically allowed windows and/or until the decoupling limit is reached. The necessary parameters, along with the corresponding allowed ranges, are collected in table 1 . Note that the lower bound of $\tan \beta$ is chosen in order to ease satisfying the constraints on the Higgs mass. The upper bound is chosen such that the phenomenology is qualitatively unchanged beyond this point. The discussion of the experimental constraints we take into account is given in the following section. $M_{A}$ could also potentially influence the relic density. It will turn out, however, that this dependence is not too strong, except for the case of the mixed wino-Higgsino. The parameters which are least relevant include the trilinear couplings and the gluino mass parameter $M_{3} .{ }^{3}$ To summarise, the most relevant parameters for our study are the wino, bino and Higgsino mass parameters $M_{2}, M_{1}$ and $\mu$, respectively, and the common sfermion mass parameter $M_{\mathrm{sf}}$. Using these inputs, the spectrum is computed using FeynHiggs $2.9 .5[23,24]$ with a top mass $m_{t}=173.2 \mathrm{GeV}$. After the initial parameter card is generated and the constraints described in section 2.2 are checked, we include one-loop corrections to the neutralino masses (see section 2.3) and take into account the running of the gauge couplings (see section 2.4).

\footnotetext{
${ }^{2}$ We leave the in-depth analysis of Higgsino or bino LSP and the sfermion co-annihilations including our detailed treatment of the Sommerfeld effect for future work. For some previous results in these cases see e.g. refs. $[9,11,15]$.

${ }^{3}$ Throughout this work we will assume that the gluinos are sufficiently heavy such that co-annihilation with the neutralino can be neglected. For a recent analysis of the relic density including gluino coannihilation see e.g. ref. [22].
} 


\begin{tabular}{|c|c|}
\hline Parameter & Range \\
\hline$M_{2}$ & $1-5 \mathrm{TeV}$ \\
$\left|M_{1}\right|$ & $M_{2}-3 M_{2}$ \\
$|\mu|$ & $M_{2}-3 M_{2}$ \\
$M_{\mathrm{sf}}$ & $1.25 M_{2}-12 \mathrm{TeV}$ \\
\hline$M_{A}$ & $0.5-10 \mathrm{TeV}$ \\
$\tan \beta$ & $5-30$ \\
\hline$\left|A_{f}\right|$ & $0-8 \mathrm{TeV}$ \\
$M_{3}$ & $3 M_{2}$ \\
\hline
\end{tabular}

Table 1. Ranges of MSSM parameters adopted for the scan, where $f$ represents all the SM quarks and leptons.

\subsection{Constraints}

We require a number of theoretical and experimental constraints to be satisfied by all the points in the scan. In this section we discuss the implementation of these constraints and comment on their relevance on limiting the ranges of the MSSM parameters, in particular in the wino-like region. All the involved quantities are computed with the use of FeynHiggs 2.9.5 and micrOMEGAs $[25,26]$.

\subsubsection{Collider and flavour constraints}

Many current collider and flavour constraints do not limit the region of the parameter space where the LSP, in our case the lightest neutralino, is at the $\mathrm{TeV}$ scale, nevertheless we include all possibly relevant constraints for completeness.

Higgs mass. We require that the light Higgs mass $m_{h^{0}}$ lies within $4 \%$ deviation of the measured central value $125.09 \pm 0.21 \pm 0.11 \mathrm{GeV}$, from the combination of ATLAS and CMS data [27]. In our numerical analysis we adopt the two-loop result for the Higgs mass which we calculate using FeynHiggs 2.9.5. Beyond $M_{\mathrm{sf}}=6-7 \mathrm{TeV}$, the allowed window is slightly $(1-2 \mathrm{GeV})$ below the estimation of the theoretical uncertainties in the Higgs mass determination in the MSSM [28], given that we do not include the resummation of logarithmic corrections arising due to the large hierarchy between the top and the stop masses. The approximate formula for the Higgs mass at one-loop level reads [29, 30]:

$$
m_{h^{0}}^{2} \simeq m_{Z}^{2} \cos ^{2} 2 \beta+\frac{3}{4 \pi^{2}} \frac{m_{t}^{4}}{v^{2}}\left[\log \frac{M_{\mathrm{sf}}^{2}}{m_{t}^{2}}+\frac{X_{t}^{2}}{M_{\mathrm{sf}}^{2}}\left(1-\frac{X_{t}^{2}}{12 M_{\mathrm{sf}}^{2}}\right)\right] .
$$

From this expression we deduce that the main implication of the Higgs mass constraint is to impose that either the stop masses are a few $\mathrm{TeV}$, or the stop mixing is large. The first condition is often satisfied in the scenarios we consider, and when not the mixing can easily be chosen such that this constraint is satisfied. Note that as $X_{t}$ does not play a significant role in the relic density computation in the wino-like region, and that for $\tan \beta \gtrsim 5, \cos 2 \beta$ 
is close to -1 , the Higgs mass constraint does not have much impact on our results. For $M_{\mathrm{sf}}>6-7 \mathrm{TeV}$ the effect of the neglected corrections to the Higgs mass could therefore be compensated by a change in $X_{t}$, leaving the relic density unaltered.

$\boldsymbol{\rho}$ parameter. We require that the value of $\Delta_{\rho}$ computed in the MSSM [31] does not exceed two standard deviations from the SM expectation [32]:

$$
\rho_{0}=1.0004 \pm 0.00024, \quad \text { therefore } \Delta_{\rho}<0.00048
$$

Since the SUSY contribution can only be large when the mass splitting in the sfermion $\mathrm{SU}(2)$ doublets is large, and in the scenario we consider all the sfermion doublets are nearly degenerate, it does not have a significant effect on our parameter space.

$\boldsymbol{b} \rightarrow \boldsymbol{s} \boldsymbol{\gamma}$. In general MSSM scenarios this branching ratio provides a strong constraint, as the contribution from broken SUSY is generically large, while the SM prediction is compatible with measurement. The experimental [33] and SM theory [34] values, with the corresponding uncertainties, we use are

$$
\begin{aligned}
& \mathcal{B}^{\exp }\left(\bar{B} \rightarrow X_{s} \gamma\right)=(3.37 \pm 0.23) \times 10^{-4} \\
& \mathcal{B}^{\mathrm{SM}}\left(\bar{B} \rightarrow X_{s} \gamma\right)=(3.15 \pm 0.23) \times 10^{-4}
\end{aligned}
$$

The SUSY contribution $\Delta \mathcal{B}\left(\bar{B} \rightarrow X_{s} \gamma\right)$ is computed with FeynHiggs and the implemented criterion reads

$$
\left[\left(\mathcal{B}^{\mathrm{SM}}\left(\bar{B} \rightarrow X_{s} \gamma\right)+\Delta \mathcal{B}\left(\bar{B} \rightarrow X_{s} \gamma\right)\right)-\mathcal{B}^{\exp }\left(\bar{B} \rightarrow X_{s} \gamma\right)\right]^{2}<\left(3 \sigma^{\exp }\right)^{2}+\left(\sigma^{\mathrm{SM}}\right)^{2}
$$

There are three classes of diagrams which contribute to $b \rightarrow s \gamma$ in the MSSM: these are diagrams involving either charged Higgs bosons, charginos or gluinos. The first always interfere constructively with the SM contribution, and decouple as the Higgs mass increases beyond the $\mathrm{TeV}$ scale. The chargino contribution can take either sign, depending on the sign of $\mu$ and $A_{t}$, but also decouples with increasing $|\mu|$ and $M_{2}$. At the scales that are relevant to this study, i.e. above $1 \mathrm{TeV}$, in general the MSSM contribution lies within the uncertainties.

$\boldsymbol{B}_{s} \rightarrow \boldsymbol{\mu}^{+} \boldsymbol{\mu}^{-}$. The correction to $B_{s} \rightarrow \mu^{+} \mu^{-}$from SUSY should also lie within the errors from the experimental measurement and the SM calculation. To this end, we check whether the result of the calculation in the MSSM [31] is consistent with the combined CMS and LHCb result, $(2.9 \pm 0.7) \times 10^{-9}$ [35]. The 3 sigma error on the experimental result is added to the uncertainty on the theoretical result in quadrature, where the updated SM prediction is $(3.56 \pm 0.30) \times 10^{-9}$, using latest values on the $B_{s}^{0}$ lifetime and relative $B_{s}^{0}$ decay width difference $[35,36]$. We note that as we consider the wino-like region with masses of the LSP of $\mathcal{O}(\mathrm{TeV})$, and masses of the heavy Higgs bosons also of $\mathcal{O}(\mathrm{TeV})$, this constraint does not have much influence on our parameter space. Another related constraint is of course the branching ratio of $B \rightarrow \tau \nu$, measured precisely at the B-factories [37, 38]. However, we do not consider this constraint as the parameter space of interest in our analysis, in particular the large masses of the charged Higgs bosons and values of $\tan \beta$, do not result in MSSM contributions beyond the combined experimental and theoretical uncertainty [39]. 
$\boldsymbol{g}_{\boldsymbol{\mu}} \mathbf{- 2}$. The experimental and SM theory values adopted for the muon anomalous magnetic moment, $a_{\mu}=\frac{g_{\mu}-2}{2}$, are given by [32],

$$
\begin{aligned}
& a_{\mu}^{\exp }=(1165920.91 \pm 0.63) \times 10^{-9} \\
& a_{\mu}^{\mathrm{SM}}=(1165918.03 \pm 0.48) \times 10^{-9}
\end{aligned}
$$

and we require that $\Delta a_{\mu}$, the MSSM contribution, satisfies

$$
-\sigma^{\exp -\mathrm{SM}}<\Delta a_{\mu}<\left(a_{\mu}^{\exp }-a_{\mu}^{\mathrm{SM}}\right)+\sigma^{\exp -\mathrm{SM}}
$$

where for the error on the difference between experimental and SM values we take

$$
\sigma^{\text {exp-SM }}=\sqrt{\left(3 \sigma^{\exp }\right)^{2}+\left(\sigma^{\mathrm{SM}}\right)^{2}}
$$

This means that we do not insist that the MSSM contribution explains the deviation between the experimental and SM theory values. Note that as the SUSY contribution is proportional to $\tan \beta$ and inversely proportional to the square of the masses of the sparticles, it is typically strongly suppressed in the region of interest where $M_{\mathrm{sf}}$ lies at the TeV scale.

\subsubsection{Theoretical constraints}

Higgs potential. Theoretical consistency demands that the scalar potential is free from charge and/or colour breaking minima (CCB). For the tree-level scalar potential in the MSSM, the corresponding criteria read [40, 41]

$$
\begin{array}{r}
A_{t}^{2}<3\left(\cos ^{2} \beta M_{A}^{2}+\frac{m_{Z}^{2}}{2} \cos 2 \beta+2 M_{\mathrm{sf}}^{2}\right), \\
A_{b(\tau)}^{2}<3\left(\sin ^{2} \beta M_{A}^{2}-\frac{m_{Z}^{2}}{2} \cos 2 \beta+2 M_{\mathrm{sf}}^{2}\right),
\end{array}
$$

One can always choose the trilinear couplings low enough such that the CCB constraint is satisfied without altering the nature of the neutralino.

s-channel resonances. Our calculation relies on the factorisation of the annihilation cross section into the short-range tree-level annihilation and the long-range potential interaction. However, this factorization does not hold in the case that the final light particles are produced through an s-channel propagator which is resonant, as such a contribution cannot be attributed to the short-distance part of the annihilation. Therefore, we need to exclude regions of parameter space where this may occur. In the MSSM this means that we need to avoid s-channel resonances through the Higgs bosons, and to be conservative we assume that the masses of the heavy Higgses lie outside the interval

$$
m_{H^{0}, A^{0}, H^{+}} \notin\left[1.7 m_{\tilde{\chi}_{1}^{0}}, 2.3 m_{\tilde{\chi}_{1}^{0}}\right] .
$$

It follows that in this work we are not in a position to study the $H$ - and $A$-funnel regions $[42,43]$. 


\subsubsection{Cosmological and direct DM detection constraints}

In choosing suitable points to calculate the Sommerfeld effect on the relic density, we insist that certain basic constraints are fulfilled. First we require that the lightest neutralino $\tilde{\chi}_{1}^{0}$ is the LSP. We further insist on compatibility with Direct Detection bounds. The details of how these conditions are imposed is described in this subsection. We choose not to include any limits coming from Indirect Detection experiments or measurements of the CMB, as although these may be relevant they are subject to large systematic uncertainties and their discussion goes beyond the scope of this work; we plan to address such constraints in the future.

Direct detection. We require that the DM-nucleon spin-independent cross section $\sigma^{\mathrm{SI}}$ is less than twice the LUX limit [44]. The theoretical prediction of this cross section within the MSSM is obtained using micrOMEGAs. The spin-independent cross section is sensitive to the Higgs exchange between the LSP and the quarks of the nucleon. The interaction with the Higgs relies on the LSP containing both gaugino and Higgsino components, and therefore this constraint is most relevant for the scenarios we study where $|\mu| \sim M_{2}$. Note that the limits of the spin-dependent cross section coming from Direct Detection experiments and neutrino signals from the Sun are always much weaker than those coming from spin-independent results for the scenarios we are interested in here.

\section{$2.3 \quad$ One-loop mass splittings}

The differences in mass between the LSP and the heavier neutralinos and charginos can have an effect on the relic density. The most relevant case is the small mass difference between the lightest chargino and neutralino state, $\tilde{\chi}_{1}^{+}$and $\tilde{\chi}_{1}^{0}$, respectively. In order to be consistent with the accuracy of the rest of the calculation we calculate these masses at one-loop. In doing so we adopt an on-shell renormalisation scheme, which is described here in brief. For further details we refer the reader to refs. [45-49].

The mass matrix in the chargino sector is renormalised via $X \rightarrow X+\delta X$, where $\delta X$ is defined by

$$
\delta X=\left(\begin{array}{cc}
\delta M_{2} & \sqrt{2} \delta\left(m_{W} s_{\beta}\right) \\
\sqrt{2} \delta\left(m_{W} c_{\beta}\right) & \delta \mu
\end{array}\right),
$$

containing the renormalisation constants (RCs) for the wino parameter $M_{2}$ and Higgsino parameter $\mu$, i.e. $\delta M_{2}$ and $\delta \mu$. In addition, the matrix $\delta X$ contains the RCs of $c_{\beta}$ and $s_{\beta}$, i.e. $\delta c_{\beta}$ and $\delta s_{\beta}$ (which can be expressed in terms of $\delta \tan \beta$ ), and of the $W$ boson mass $m_{W}, \delta m_{W}$. Definitions of and expressions for $\delta \tan \beta$ and $\delta m_{W}$ can be found in ref. [49]. The neutralino mass matrix, $Y$, is renormalised in a similar manner via $Y \rightarrow Y+\delta Y$, where $\delta Y$ is defined in analogy to $\delta X$ in eq. (2.17) and further contains RC of the bino parameter $M_{1}, \delta M_{1}$. In the on-shell scheme, we must fix the RCs $\delta M_{1}, \delta M_{2}$ and $\delta \mu$ (as in e.g. ref. [46]) by requiring that three out of the total six physical masses of the charginos and neutralinos satisfy on-shell conditions, i.e. that the tree-level masses, $m_{\tilde{\chi}_{i}}$, coincide with the one-loop renormalised masses, $M_{\tilde{\chi}_{i}}=m_{\tilde{\chi}_{i}}+\Delta m_{\tilde{\chi}_{i}}$,

$$
\Delta m_{\tilde{\chi}_{i}} \equiv-\frac{m_{\tilde{\chi}_{i}}}{2} \operatorname{Re}\left[\hat{\Sigma}_{i i}^{L}\left(m_{\tilde{\chi}_{i}}^{2}\right)+\hat{\Sigma}_{i i}^{R}\left(m_{\tilde{\chi}_{i}}^{2}\right)\right]-\frac{1}{2} \operatorname{Re}\left[\hat{\Sigma}_{i i}^{S L}\left(m_{\tilde{\chi}_{i}}^{2}\right)+\hat{\Sigma}_{i i}^{S R}\left(m_{\tilde{\chi}_{i}}^{2}\right)\right]=0 .
$$




\begin{tabular}{|c|c|}
\hline Scenario & Particles on shell \\
\hline$M_{2}<\left|M_{1}\right|<|\mu|$ & $\tilde{\chi}_{1}^{+}, \tilde{\chi}_{2}^{0}, \tilde{\chi}_{3}^{0}$ \\
$M_{2}<|\mu|<\left|M_{1}\right|$ & $\tilde{\chi}_{1}^{+}, \tilde{\chi}_{2}^{0}, \tilde{\chi}_{4}^{0}$ \\
\hline
\end{tabular}

Table 2. Choice of particles whose masses are required to be on shell for the various scenarios corresponding to the possible orderings of $M_{1}, M_{2}$ and $\mu$ that we consider.

Note that we define the coefficients $\hat{\Sigma}_{i j}^{L / R}\left(p^{2}\right)$ and $\hat{\Sigma}_{i j}^{S L / S R}\left(p^{2}\right)$ of the self energy via

$$
\hat{\Sigma}_{i j}\left(p^{2}\right)=\not p P_{L} \hat{\Sigma}_{i j}^{L}\left(p^{2}\right)+\not p P_{R} \hat{\Sigma}_{i j}^{R}\left(p^{2}\right)+P_{L} \hat{\Sigma}_{i j}^{S L}\left(p^{2}\right)+P_{R} \hat{\Sigma}_{i j}^{S R}\left(p^{2}\right) .
$$

The left- and right-handed vector and scalar coefficients, $\hat{\Sigma}_{i j}^{L / R}\left(p^{2}\right)$ and $\hat{\Sigma}_{i j}^{S L / S R}\left(p^{2}\right)$ of the renormalised self-energy are defined analogously. Expressions for the renormalised selfenergies can be found in e.g. ref. [49]. The mass shifts for the remaining three chargino and neutralino masses are therefore given by $\Delta m_{\tilde{\chi}_{i}^{ \pm}}$and $\Delta m_{\tilde{\chi}_{j}^{0}}$ in eq. (2.18). For the calculation of these mass shifts we used the program FeynArts $[50,51]$, together with the packages FormCalc [52] and LoopTools [52], using the model files presented in ref. [53].

The choice of which masses should be chosen on shell is non-trivial, as certain choices can lead to unphysical divergences when e.g. $\left|M_{1}\right|=M_{2}$ or $|\mu|=M_{2}$, and we follow the prescription discussed in refs. $[48,54]$ as follows to avoid this situation as far as possible. We therefore employ the NNC scheme, that is, two neutralinos and one chargino are chosen on-shell, of which the chargino should be wino-like, and the neutralinos should be bino and Higgsino-like. Note however that there is an ambiguity here given that there are two Higgsino-like neutralinos. In this work we are particularly interested in the region where the neutralino has a large wino component, i.e. $M_{2}<\left|M_{1}\right|,|\mu|$, and may in addition contain a sizeable bino or Higgsino component. We therefore find that in order to obtain results free from scheme-dependent divergences, the choice of particles whose masses are required to be on shell should be made as in table 2. This corresponds to the Higgsino closer in mass to the wino being on shell. Note that when all three parameters are very close $(<0.1 \%$ splittings) the situation may arise that the ordering of the neutralinos changes, and one should exercise caution in these regions. This has been accounted for in the code.

\subsection{Running couplings}

Due to the multi-scale nature of the considered problem, the running of the coupling constants has to be treated consistently. In different parts of the calculation the couplings should be taken at a different energy scale $Q$, in particular $Q=m_{Z}$ for the potential interactions, $Q=m_{\mathrm{LSP}}$ for the mass splittings in the neutralino/chargino sector and $Q=$ $2 m_{\mathrm{LSP}}$ for the short-range annihilations.

We perform the running in the unbroken $\mathrm{SU}(2)_{L} \times \mathrm{U}(1)_{Y}$ theory, since most of the running occurs above the electroweak scale. The starting values of the $\mathrm{SU}(2)_{L}$ and $\mathrm{U}(1)$ couplings at $Q=m_{Z}$ are taken as $\alpha_{2}\left(m_{Z}\right)=0.034723$ and $\alpha_{1}\left(m_{Z}\right)=0.009986$, respectively. Since the short-range annihilation is evaluated at tree-level, we run the couplings to 


\begin{tabular}{|c|cc|cc|cc|}
\hline \multirow{3}{*}{ Particles } & \multicolumn{2}{|c|}{$\operatorname{Tr}\left[T_{A}^{(i) 2}\right]$} & \multicolumn{2}{|c|}{$\sum_{f} \operatorname{Tr}\left[T_{f}^{(i) 2}\right]$} & \multicolumn{2}{|c|}{$\sum_{s} \operatorname{Tr}\left[T_{s}^{(i) 2}\right]$} \\
& $\mathrm{U}(1)_{Y}$ & $\mathrm{SU}(2)_{L}$ & $\mathrm{U}(1)_{Y}$ & $\mathrm{SU}(2)_{L}$ & $\mathrm{U}(1)_{Y}$ & $\mathrm{SU}(2)_{L}$ \\
\hline $\mathrm{SM}$ & 0 & 2 & 10 & 6 & $\frac{1}{2}$ & $\frac{1}{2}$ \\
\hline$\Phi_{2}$ & 0 & 0 & 0 & 0 & $\frac{1}{2}$ & $\frac{1}{2}$ \\
\hline$\tilde{B}$ & 0 & 0 & 0 & 0 & 0 & 0 \\
\hline$\tilde{W}$ & 0 & 0 & 0 & 2 & 0 & 0 \\
\hline$\tilde{H}$ & 0 & 0 & 1 & 1 & 0 & 0 \\
\hline
\end{tabular}

Table 3. Contributions to the $\mathrm{U}(1)_{Y}$ and $\mathrm{SU}(2)_{L}$ beta functions.

$Q=2 m_{\mathrm{LSP}}$ with the one-loop renormalisation group equation. In the computation of the one-loop mass splittings discussed above, the couplings are evaluated at $Q=m_{\mathrm{LSP}}$. The energy range from $m_{Z}$ to $2 m_{\mathrm{LSP}}$ that we are interested in can be divided into five regions ${ }^{4}$ where the beta functions are constant, delimited by the scales $M_{A},\left|M_{1}\right|, M_{2},|\mu|$, at which we decouple respectively the heavy Higgs doublet $\Phi_{2}$, the bino, the wino, and the Higgsinos.

At the required level of accuracy there are no threshold effects to be considered and the leading order beta function $\beta_{0, i}$ at a scale $Q$ is given by

$$
\beta_{0, i}=\frac{11}{3} \operatorname{Tr}\left[T_{A}^{(i) 2}\right]-\frac{2}{3} \sum_{f} \operatorname{Tr}\left[T_{f}^{(i) 2}\right]-\frac{1}{3} \sum_{s} \operatorname{Tr}\left[T_{s}^{(i) 2}\right],
$$

where $T_{R}^{(i)}$ are the generators of the group $i$ in the representation $R$ and the three terms correspond respectively to gauge bosons (always in the adjoint representation $A$ ), fermions, and scalars. The sums extend only to particles with mass smaller than $Q$, and the contributions are listed in table 3 .

\subsection{Annihilation matrix implementation}

The rate at which neutralinos and charginos annihilate into the (light) standard model particles in the early Universe is a necessary input for the calculation of the present-day amount of dark matter. For a given two-particle state $\tilde{\chi}_{i} \tilde{\chi}_{j} \equiv[\tilde{\chi} \tilde{\chi}]_{a}$ formed out of two neutralino or chargino species, the annihilation rate including long-distance Sommerfeld corrections can be parametrised as [14]

$$
\sigma^{[\tilde{\chi} \tilde{\chi}]_{a} \rightarrow \operatorname{light}} v_{\mathrm{rel}}=S_{a}\left[\hat{f}_{h}\left({ }^{1} S_{0}\right)\right] \hat{f}_{a a}\left({ }^{1} S_{0}\right)+S_{a}\left[\hat{f}_{h}\left({ }^{3} S_{1}\right)\right] 3 \hat{f}_{a a}\left({ }^{3} S_{1}\right)
$$

\footnotetext{
${ }^{4}$ We neglect the sfermions' contribution to the beta functions, when the sfermion mass lies between $m_{\mathrm{LSP}}$ and $2 m_{\mathrm{LSP}}$. The error introduced in this way is small: for a $2.5 \mathrm{TeV}$ LSP the total running of $\alpha_{2}$ from $m_{Z}$ up to $2 m_{\mathrm{LSP}}$ is around $5-6 \%$, and the maximum contribution from sfermions (when they are all decoupled at their smallest allowed mass $1.25 \mathrm{~m}_{\mathrm{LSP}}$ ) is only $0.4 \%$.
} 


$$
\begin{aligned}
& +\frac{\vec{p}_{a}^{2}}{M_{a}^{2}}\left(S_{a}\left[\hat{g}_{\kappa}\left({ }^{1} S_{0}\right)\right] \hat{g}_{a a}\left({ }^{1} S_{0}\right)+S_{a}\left[\hat{g}_{\kappa}\left({ }^{3} S_{1}\right)\right] 3 \hat{g}_{a a}\left({ }^{3} S_{1}\right)\right. \\
& \left.+S_{a}\left[\frac{\hat{f}\left({ }^{1} P_{1}\right)}{M^{2}}\right] \hat{f}_{a a}\left({ }^{1} P_{1}\right)+S_{a}\left[\frac{\hat{f}\left({ }^{3} P_{\mathcal{J}}\right)}{M^{2}}\right] \hat{f}_{a a}\left({ }^{3} P_{\mathcal{J}}\right)\right),
\end{aligned}
$$

up to higher orders in $\vec{p}_{a}^{2}=2 \mu_{i j}\left(\sqrt{s}-M_{a}\right)+\ldots$, the relative momentum of the annihilating particles in their centre-of-mass frame, with $M_{a}, \mu_{a}$ the total and reduced mass, respectively, of the two-particle state. The quantities $\hat{f}_{a b}\left({ }^{2 S+1} L_{J}\right), \hat{g}_{a b}\left({ }^{2 S+1} L_{J}\right), \ldots$ are the absorptive part of the Wilson coefficients of local four-fermion operators which reproduce the short-distance annihilation of the chargino and neutralino pairs into SM and light Higgs final states in the non-relativistic EFT framework [12-14]. They were determined by matching the tree-level MSSM amplitudes for the process $[\tilde{\chi} \tilde{\chi}]_{a} \rightarrow X_{A} X_{B} \rightarrow[\tilde{\chi} \tilde{\chi}]_{b}$ with SM and Higgs intermediate states $X_{A} X_{B}$ in refs. [12, 13]. ${ }^{5}$ The definition of the various Wilson coefficients appearing in eq. (2.21) can be found in ref. [14]. The Sommerfeld factors $S_{a}[\ldots]$ in eq. (2.21) account for the long-distance interactions of the two-particle states prior to the short-distance annihilation. Details on the computation of these factors are given below. The tree-level annihilation rate with no long-distance corrections is readily recovered by setting all the Sommerfeld factors in eq. (2.21) to one. The tree-level annihilation cross section thus obtained depends only on the diagonal entry of the Wilson coefficients corresponding to channel $[\tilde{\chi} \tilde{\chi}]_{a}$, i.e. $\hat{f}_{a a}\left({ }^{2 S+1} L_{J}\right), \hat{g}_{a a}\left({ }^{2 S+1} L_{J}\right), \ldots$ As shown in eq. (2.28) below, the computation of the Sommerfeld factors also requires knowledge of the off-diagonal terms, $\hat{f}_{a b}\left({ }^{2 S+1} L_{J}\right), \hat{g}_{a b}\left({ }^{2 S+1} L_{J}\right), \ldots$, with $a \neq b$, since the interference of loop diagrams where the two-particle states that undergo short-distance annihilation are different are accounted for in the Sommerfeld-corrected cross section.

A word on the notation for labelling the two-particle states is relevant here. The two-particle states $\tilde{\chi}_{i} \tilde{\chi}_{j}$ formed out of charginos and neutralinos are denoted by a single label $a=1, \ldots N_{|Q|}$, where $N_{|Q|}$ is the total number of states (channels) for each electriccharge sector, $|Q|=0,1,2$, corresponding to neutral $\left(\tilde{\chi}^{0} \tilde{\chi}^{0}, \tilde{\chi}^{+} \tilde{\chi}^{-}\right)$, single-charged $\left(\tilde{\chi}^{0} \tilde{\chi}^{ \pm}\right)$ and double-charged $\left(\tilde{\chi}^{ \pm} \tilde{\chi}^{\mp}\right)$ sectors. If all four neutralinos and the two charginos are considered, in the charge- 0 sector the single label runs over the 14 different states

$$
\tilde{\chi}_{1}^{0} \tilde{\chi}_{1}^{0}, \tilde{\chi}_{1}^{0} \tilde{\chi}_{2}^{0}, \tilde{\chi}_{1}^{0} \tilde{\chi}_{3}^{0}, \ldots, \tilde{\chi}_{3}^{0} \tilde{\chi}_{4}^{0}, \tilde{\chi}_{4}^{0} \tilde{\chi}_{4}^{0}, \tilde{\chi}_{1}^{+} \tilde{\chi}_{1}^{-}, \tilde{\chi}_{1}^{+} \tilde{\chi}_{2}^{-}, \tilde{\chi}_{2}^{+} \tilde{\chi}_{1}^{-}, \tilde{\chi}_{2}^{+} \tilde{\chi}_{2}^{+}
$$

whereas in the charge \pm 1 sectors we have 8 channels each,

$$
\tilde{\chi}_{1}^{0} \tilde{\chi}_{1}^{ \pm}, \tilde{\chi}_{1}^{0} \tilde{\chi}_{2}^{ \pm}, \ldots, \tilde{\chi}_{4}^{0} \tilde{\chi}_{1}^{ \pm}, \tilde{\chi}_{4}^{0} \tilde{\chi}_{2}^{ \pm}
$$

and just three each in the charge \pm 2 sectors,

$$
\tilde{\chi}_{1}^{ \pm} \tilde{\chi}_{1}^{ \pm}, \tilde{\chi}_{1}^{ \pm} \tilde{\chi}_{2}^{ \pm}, \tilde{\chi}_{2}^{ \pm} \tilde{\chi}_{2}^{ \pm}
$$

The coefficients $\hat{f}_{a b}\left({ }^{2 S+1} L_{J}\right)$ for each partial wave can then be considered as the entries of a matrix whose dimension is equal to the number of channels in each sector. Since the

\footnotetext{
${ }^{5}$ We have dropped an upper index " $\chi \chi \rightarrow \chi \chi$ " used in refs. [12, 13] for the notation of the Wilson coefficients.
} 
coefficients $\hat{f}_{a b}\left({ }^{2 S+1} L_{J}\right)$ have the property $\hat{f}_{b a}\left({ }^{2 S+1} L_{J}\right)=\left[\hat{f}_{a b}\left({ }^{2 S+1} L_{J}\right)\right]^{*}$, such annihilation matrices turn out to be hermitian. The computation of each of the annihilation matrices appearing in the annihilation cross section formula (2.21), requires the evaluation of 105 , $2 \times 36$ and $2 \times 6$ distinct entries for neutral, single- and double-charged sectors, respectively. Ten of such matrices are needed for a complete calculation of the Sommerfeld-corrected annihilation cross section including $\mathcal{O}\left(v^{2}\right)$ corrections (see ref. [14] for details on this), making up a total number of 1890 independent entries. In the CP-conserving case, the annihilation cross sections of the charged-conjugated sectors, $\tilde{\chi}^{0} \tilde{\chi}^{+}$and $\tilde{\chi}^{0} \tilde{\chi}^{-}, \tilde{\chi}^{+} \tilde{\chi}^{+}$and $\tilde{\chi}^{-} \tilde{\chi}^{-}$, become equal, and the number of independent annihilation matrix entries is reduced to 1470 .

A code to obtain the analytic results for the entries of the annihilation matrices at $\mathcal{O}\left(\alpha_{2}^{2}\right)$ in the MSSM has been developed following the conventions and recipes of refs. [12, 13, 55]. The expressions account for the sum of all possible $X_{A} X_{B}$ exclusive states with $X_{A / B}$ being a SM particle (including the light Higgs) or heavy MSSM Higgs (the mass of the state $X_{A} X_{B}$ must however be smaller than $\left.2 m_{\mathrm{LSP}}\right) .{ }^{6}$ For the neutral, single- and doublecharged sectors, the number of exclusive final states is 31,16 and 3 , respectively, including the possible heavy Higgs final states; a complete list can be found in appendix A of ref. [12]. Despite coming from the product of tree-level amplitudes, the analytic expressions for the Wilson coefficients are very large, which is traced back to the fact that there are several diagrams with different topologies and/or virtual intermediate particles contributing to a given exclusive state, and because of the non-relativistic expansion performed. Recall as well that we keep the general dependence on all MSSM parameters in the coefficients. The numerical evaluation of all matrix entries for a given MSSM parameter set is done using precompiled functions within MATHEMATICA, taking on average approximately 300 sec of CPU time. If only the annihilation matrices necessary for the leading-order cross section, $\hat{f}_{a b}\left({ }^{1} S_{0}\right)$ and $\hat{f}_{a b}\left({ }^{3} S_{1}\right)$, are evaluated, the cost in CPU time reduces to less than 40 sec per model.

We should mention here a modification of a part of the analytic expressions for the Wilson coefficients given in refs. $[12,13]$ that we have implemented in the present code. The Wilson coefficients obtained in refs. $[12,13]$ describe $[\tilde{\chi} \tilde{\chi}]_{a} \rightarrow[\tilde{\chi} \tilde{\chi}]_{b}$ annihilation amplitudes expanded in powers of $\sqrt{s}-M$, where $M \equiv\left(M_{a}+M_{b}\right) / 2$ is the average of the masses of the two-particle states taking place in the short-distance part of the annihilation process (for diagonal reactions, $a=b$, this is just an expansion around the $[\tilde{\chi} \tilde{\chi}]_{a}$ threshold). When the annihilation proceeds through s-channel boson exchange, such an expansion implies for the boson propagator (with generic mass $m_{\phi}$ ) that

$$
\frac{1}{s-m_{\phi}^{2}}=\frac{1}{M^{2}-m_{\phi}^{2}}\left(1-\frac{2 M(\sqrt{s}-M)}{M^{2}-m_{\phi}^{2}}+\ldots\right)
$$

up to linear terms in $\sqrt{s}-M$. The first term on the right-hand side of eq. (2.25) contributes to the leading-order Wilson coefficients, whereas the second goes to the $S$-wave $v^{2}$-suppressed ones. For heavy Higgs exchange, the following problem may arise: once

\footnotetext{
${ }^{6}$ Strictly speaking, one should allow for $m_{X_{A} X_{B}}<M_{I}$ when we are dealing with the co-annihilation cross section of the external 2-particle state $I$, but this would require having a different set of annihilation matrices for each co-annihilation channel, which is impractical.
} 
radiative corrections are included, any (virtual) states $a, b$ can participate in the shortdistance part, such that we can find a situation where $M$ gets very close to the Higgs mass $m_{\phi} \approx M_{A}$, producing arbitrarily large contributions in the right-hand side of eq. (2.25). Those resonance contributions are spurious, since the annihilating cross section of the external state $[\tilde{\chi} \tilde{\chi}]_{I}$ in the non-relativistic regime should be expanded for energies close to the mass of that state, i.e. around $\sqrt{s}=M_{I}$, which produces terms from s-channel contributions proportional to $1 /\left(M_{I}^{2}-m_{\phi}^{2}\right)$ instead of those in eq. (2.25). For the relevant co-annihilation channels, the latter terms cannot become resonant in our analysis because we have explicitly excluded Higgs masses inside the range $\left[1.7 m_{\tilde{\chi}_{1}^{0}}, 2.3 m_{\tilde{\chi}_{1}^{0}}\right]$, see eq. (2.16). Therefore, the problem of spurious resonances is absent if we have a set of annihilation matrices for each co-annihilation channel $I$ where the s-channel propagators have been expanded around $\sqrt{s}=M_{I}$. In practice, that solution is unfeasible, since the number of co-annihilations channels in a mixed scenario can be rather large and evaluating several annihilation matrices would increase the required CPU time beyond reasonable limits. We can adopt, however, another solution that avoids the occurrence of spurious resonances in s-channel propagators that only requires minimal changes in the Wilson coefficients obtained in refs. [12, 13]. It amounts to modifying the expanded s-channel propagators from the Wilson coefficients such that they correspond to their expansion around $\sqrt{s}=2 m_{\tilde{\chi}_{1}^{0}}$, regardless of which is the external co-annihilating state. We note that since the relevant channels that are included in the long-distance radiative corrections are very close in mass (see next section), the differences between the annihilation amplitudes expanded around $2 m_{\tilde{\chi}_{1}^{0}}$ or around any of the other masses of the co-annihilating states are in any case negligible, and the suggested prescription is a very good approximation. The necessary modifications can be immediately read off by rewriting the right-hand side of eq. (2.25) using $M=2 m_{\tilde{\chi}_{1}^{0}}+\left(M-2 m_{\tilde{\chi}_{1}^{0}}\right)$ :

$$
\begin{aligned}
& \frac{1}{M^{2}-m_{\phi}^{2}}\left(1-\frac{2 M(\sqrt{s}-M)}{M^{2}-m_{\phi}^{2}}\right) \\
& \quad=\frac{1}{4 m_{\tilde{\chi}_{1}^{0}}^{2}-m_{\phi}^{2}}\left(1-\frac{4 m_{\tilde{\chi}_{1}^{0}}\left(M-2 m_{\tilde{\chi}_{1}^{0}}\right)}{4 m_{\tilde{\chi}_{1}^{0}}^{2}-m_{\phi}^{2}}-\frac{4 m_{\tilde{\chi}_{1}^{0}}^{2}(\sqrt{s}-M)}{4 m_{\tilde{\chi}_{1}^{0}}^{2}-m_{\phi}^{2}}\right),
\end{aligned}
$$

where we have dropped terms of second order in the small quantities $\left(M-2 m_{\tilde{\chi}_{1}^{0}}\right)$ and $(\sqrt{s}-M)$. We notice that the dependence on $M$ cancels out in the second line of eq. (2.26), and the resulting expression matches the expansion of the Higgs propagator $1 /\left(s-m_{\phi}^{2}\right)$ around $\sqrt{s}=2 m_{\tilde{\chi}_{1}^{0}}$. The replacements that have to be performed in the Wilson coefficients of refs. $[12,13]$ thus read:

$$
\begin{aligned}
& \text { LO Wilson coeffs.: } \quad \frac{1}{M^{2}-m_{\phi}^{2}} \longrightarrow \frac{1}{4 m_{\tilde{\chi}_{1}^{0}}^{2}-m_{\phi}^{2}}\left(1-\frac{4 m_{\tilde{\chi}_{1}^{0}}\left(M-2 m_{\tilde{\chi}_{1}^{0}}\right)}{4 m_{\tilde{\chi}_{1}^{0}}^{2}-m_{\phi}^{2}}\right) \\
& v^{2} \text { Wilson coeffs.: } \quad \frac{1}{M^{2}-m_{\phi}^{2}} \longrightarrow \frac{1}{4 m_{\tilde{\chi}_{1}^{0}}^{2}-m_{\phi}^{2}}, \\
& \frac{2 M}{\left(M^{2}-m_{\phi}^{2}\right)^{2}} \longrightarrow \frac{4 m_{\tilde{\chi}_{1}^{0}}}{\left(4 m_{\tilde{\chi}_{1}^{0}}^{2}-m_{\phi}^{2}\right)^{2}} \text {. }
\end{aligned}
$$


The factor of $2 M$ in front of the square of a scalar propagator gets replaced by $4 m_{\tilde{\chi}_{1}^{0}}$ in the $S$-wave $v^{2}$-suppressed Wilson coefficients to get exactly the form in the right-hand side of eq. (2.26), though the difference between both expressions is formally of higher order. In self-energy contributions, the replacement (2.27) in the scalar propagator of LO Wilson coefficients produces an $\mathcal{O}\left(v^{4}\right)$ term from the product of the right and left s-channel propagators in the diagram, which is consistently dropped in our code in order to keep the expansion of Wilson coefficients to $\mathcal{O}\left(v^{2}\right)$ everywhere.

\subsection{Sommerfeld-corrected cross section}

The annihilation cross sections for the processes $[\chi \chi]_{a}=\chi_{i} \chi_{j} \rightarrow X$, eq. (2.21), are computed by multiplying every term in the partial wave expansion of the Born cross section by its specific Sommerfeld factor

$$
S_{a}\left[\hat{f}\left({ }^{2 S+1} L_{J}\right)\right]=\frac{\left[\psi_{c a}^{(L, S)}\right]^{*} \hat{f}_{b c}\left({ }^{2 S+1} L_{J}\right) \psi_{b a}^{(L, S)}}{\left.\hat{f}_{a a}\left({ }^{2 S+1} L_{J}\right)\right|_{\mathrm{LO}}} .
$$

When the Sommerfeld factors are neglected, eq. (2.21) reproduces the Born annihilation cross section including $\mathcal{O}\left(v^{2}\right)$ terms. The Sommerfeld factors are computed by solving the Schrödinger equation for a system of coupled two-particle states with the leading-order Yukawa and Coulomb potentials generated by the exchange of electroweak gauge bosons, Higgs bosons ${ }^{7}$ and the photon. For further details including notation, we refer to ref. [14].

The calculation can be done separately in the sectors of two-particle states with different electric charge $0, \pm 1, \pm 2$. Since we restrict ourselves to the CP-conserving MSSM, the annihilation cross sections for the negatively charged two-particle states are identical to the corresponding positively charged ones, and do not have to be calculated explicitly.

In every charge-sector, the Sommerfeld factors are computed for all two-particle states with mass less than $1.2 \times 2 m_{\mathrm{LSP}}$ unless the number of such states is larger than four, in which case the four lightest two-particle states are selected. For the other, heavier twoparticle states, we employ the Born cross sections. Furthermore, in the computation of the Sommerfeld factor we include the light states (at most four) exactly in the solution of the Schrödinger equation and the others approximately in the last loop near the annihilation vertex as described in [14]. The mass cut at $1.2 \times 2 m_{\mathrm{LSP}}$ is motivated by the fact that heavier states are either strongly Boltzmann-suppressed and irrelevant for freeze-out or they are sufficiently off-shell within the ladder diagrams to not contribute substantially to the Sommerfeld effect of the lighter states. The restriction to at most four light states is motivated by CPU considerations, since the time needed for the matrix Schrödinger equation solution increases rapidly with the number of stated treated exactly. The restriction is certainly sufficient for models close to the pure-wino case, when the degenerate states are $\tilde{\chi}_{1}^{0} \tilde{\chi}_{1}^{0}$, $\tilde{\chi}_{1}^{+} \tilde{\chi}_{1}^{-}$in the neutral sector, and $\tilde{\chi}_{1}^{0} \tilde{\chi}_{1}^{+}, \tilde{\chi}_{1}^{+} \tilde{\chi}_{1}^{+}$in the charge- 1 and and charge-2 sectors, respectively. When the LSP acquires a substantial Higgsino or bino component, the number of degenerate states increases and may exceed four in the neutral and charge- 1 sector. An example of a strongly mixed wino-Higgsino LSP model has been analysed in ref. [15], which

\footnotetext{
${ }^{7}$ In practice, we include the Higgs-exchange potential only when the Higgs mass is less than $m_{\mathrm{LSP}} / 2$.
} 
demonstrated that in this case the effect of the additional states is accurately reproduced by the approximate treatment in the last loop before the annihilation. In the analysis of strongly mixed wino-Higgsino LSP models with a nearly decoupled bino discussed below, all possible 10 neutral states fall below the mass cut $1.2 \times 2 m_{\mathrm{LSP}}$ in much of the interesting region. We checked on a subset of 1575 analysed model points that the relic density is always accurately reproduced by the approximate treatment. The largest difference we find is $4 \%$, but it is below $1 \%$ in $96 \%$ of these points, and most of the times closer to the permille level. In any case, this is not a restriction, since the code can always be run with the full set of states treated exactly, at the expense of an increase in CPU time of about a factor of ten.

The Sommerfeld factors are computed from the asymptotic behaviour at $r \rightarrow \infty$ of radial solutions of the Schrödinger equation with boundary conditions near the origin. In practice, evolution of the differential equation system to large $r$ is costly, and a finite value of $r_{\infty}$ must be chosen. We determine this value by requiring that the Sommerfeld factor changes by less than $0.3 \%$, when $r_{\infty}$ is doubled. This accuracy is often difficult to achieve for very small velocities $v$, defined by $E=m_{\mathrm{LSP}} v^{2}=\sqrt{s}-2 m_{\mathrm{LSP}}$ or near values, where new two-particle channels with mass above $2 m_{\mathrm{LSP}}$ open, especially for $\tilde{\chi}^{+} \tilde{\chi}^{-}$states which experience the long-range Coulomb interaction. Hence we fix $x_{\infty}=r_{\infty} /\left(m_{\mathrm{LSP}} v\right)$ to 20 (50), when $v<0.03$ (within 0.0002 of a threshold). This can lead to local inaccuracies of several percent. However, we find that the deviation from the exact result is oscillatory, and mostly averages out in the thermal average. Once again, this treatment is not necessitated by a limitation of the code but a convenience, since one can set always $x_{\infty}$ to larger values if needed.

We generate tables of annihilation cross sections $\left(\sigma v_{\text {rel }}\right)_{a}$ of two-particle states with on average around 50 velocity points chosen adaptively from $10^{-4} \ldots 1$ with more sampling points near thresholds and the characteristic velocities near the freeze-out temperature. We interpolate these functions and compute the thermally averaged effective cross section $\left\langle\sigma_{\text {eff }} v\right\rangle$, summed over all co-annihilating two-particle states for around 60 suitably chosen values of $x=m_{\mathrm{LSP}} / T$ between 1 and $10^{8}$. This table is interpolated and the interpolating function is employed in the Boltzmann equation

$$
\frac{d Y}{d x}=-\sqrt{\frac{\pi}{45 G}} \frac{g_{*}^{1 / 2} m_{1}}{x^{2}}\left\langle\sigma_{\mathrm{eff}} v\right\rangle\left(Y^{2}-Y_{\mathrm{eq}}^{2}\right)
$$

for $Y=n / s$. Here $G$ is the gravitational constant, and the parameter $g_{*}^{1 / 2}$ is defined in the standard way as

$$
g_{*}^{1 / 2}=\frac{h_{\mathrm{eff}}}{g_{\mathrm{eff}}^{1 / 2}}\left(1+\frac{T}{3 h_{\mathrm{eff}}} \frac{d h_{\mathrm{eff}}}{d T}\right)
$$

in terms of the effective degrees of freedom $g_{\text {eff }}$ and $h_{\text {eff }}$ of the energy and entropy densities:

$$
\rho=g_{\mathrm{eff}}(T) \frac{\pi^{2}}{30} T^{4}, \quad s=h_{\mathrm{eff}}(T) \frac{2 \pi^{2}}{45} T^{3} .
$$

For $g_{*}^{1 / 2}(T)$ and $h_{\text {eff }}(T)$ we use the values derived in ref. [56], which can be found conveniently tabulated as a function of temperature among the package files of the automated 
programs DarkSUSY [57] and micrOMEGAs [25, 26]. Other numerical values needed for the computation of the relic density are $T_{0}=2.7255 \mathrm{~K}$ and $\rho_{\text {crit }}=1.05368 \times 10^{-5} h^{2} \mathrm{GeV} \mathrm{cm}^{-3}$, both taken from ref. [58].

Given the annihilation matrices, the calculation of all Sommerfeld factors, cross section tables, thermal averages and, finally, the evolution of the Boltzmann equation through freeze-out takes about $400 \mathrm{sec}$ of CPU time, leading to a total computation time (including the evaulation of the annihilation matrices) of somewhat above $10 \mathrm{~min}$ per MSSM parameter point.

\section{Analysis}

The departure from the pure-wino limit can be obtained by lowering the sfermion masses and/or introducing non-negligible Higgsino or bino fractions of the lightest neutralino. Therefore we organise the analysis and results in three parts: effect of the sfermion masses (section 3.1), Higgsino admixture (section 3.2) and bino admixture (section 3.4). The residual dependence on remaining parameters is discussed in section 3.5.

\subsection{Impact of sfermions}

The role played by the sfermions in the production of the thermal neutralino relic density is threefold: i) they appear in the t- and u-channel annihilation into SM fermions, ii) they introduce corrections to the neutralino and chargino masses indirectly, via loop effects, and iii) if light enough, they can contribute to the effective annihilation cross section through additional co-annihilation channels. The last of these is beyond the scope of this work and we leave a detailed analysis of general sfermion co-annihilation regions with the inclusion of the Sommerfeld enhancement for future work. Therefore in our results for the perturbative and Sommerfeld corrected relic density, shown in figure 1 and the ratio of these results shown in figure 2, we require that all the sfermions are at least $25 \%$ heavier than the LSP. ${ }^{8}$ From points $i$ ) and ii), the indirect effect of changing the spectrum is sub-dominant, even for the regions of parameter space where the Sommerfeld effect exhibits a resonance and where the resulting cross section is extremely sensitive to the mass difference between $\tilde{\chi}_{1}^{ \pm}$and $\tilde{\chi}_{1}^{0}$. The reason is that the main contribution to this quantity comes from loops involving gauge bosons and the ones with sfermions are suppressed by their large masses.

The direct impact is on the other hand quite important. Decoupled sfermions mean that the only contributions to the effective co-annihilation cross section from the processes with SM fermion final states arise due to the s-channel annihilation through gauge or Higgs bosons. When the sfermions become lighter the t- and u-channel processes start to be non-negligible. This is especially relevant for the co-annihilation channels, while the direct LSP annihilation to SM fermions is helicity or p-wave suppressed. These t- and $\mathrm{u}$-channel diagrams involving sfermions interfere destructively with the s-channel gauge boson exchange effectively lowering the co-annihilation cross section [59].

\footnotetext{
${ }^{8}$ Note that the horizontal axis of most of our plots is chosen to be $M_{2}$, which in general lies within a few $\mathrm{GeV}$ of $m_{\mathrm{LSP}}$.
} 

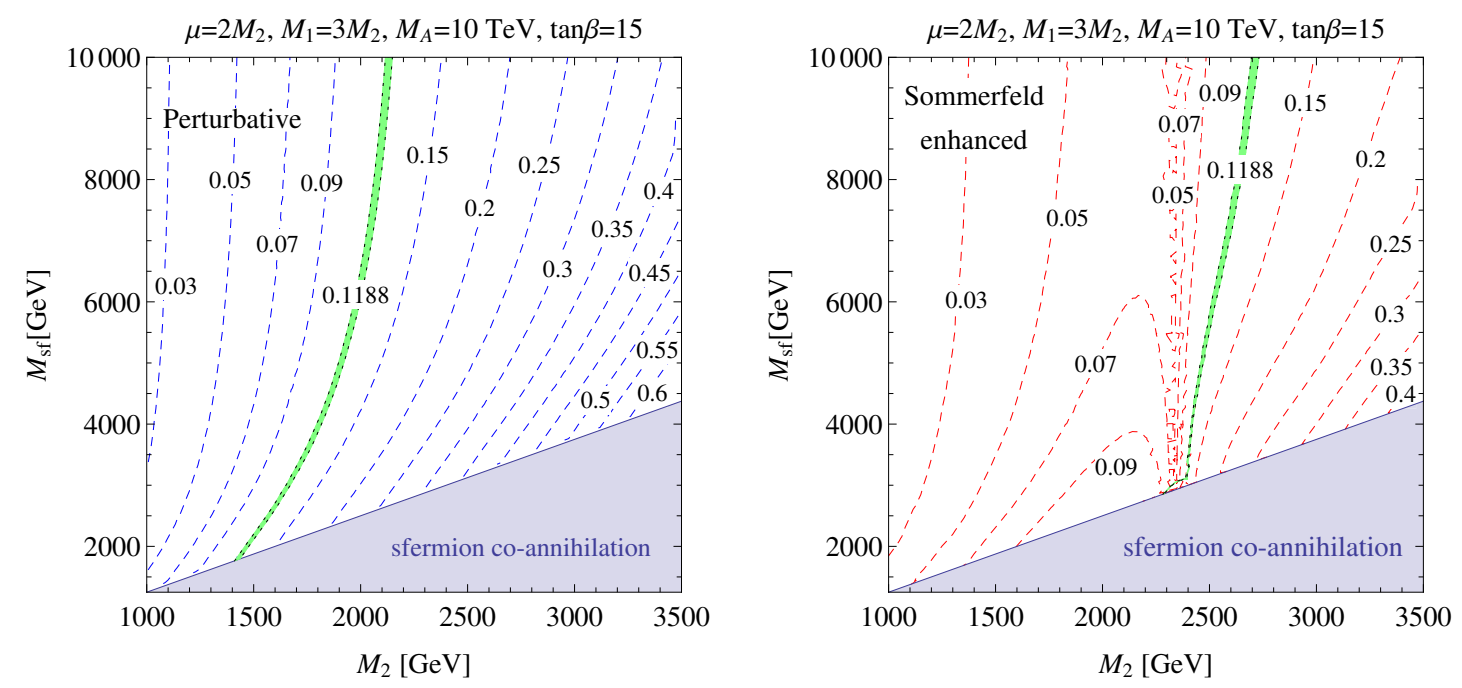

Figure 1. The contours of constant relic density: perturbative (left) and Sommerfeld enhanced (right). The (green) bands show the region within $2 \sigma$ of the observed dark matter abundance. The grey area indicates the region in parameter space where the co-annihilations with sfermions are potentially relevant and which is not studied in this work. Other parameters are as indicated, with $A_{i}=8 \mathrm{TeV}$ and $X_{t}$ is fixed by the measured Higgs mass.

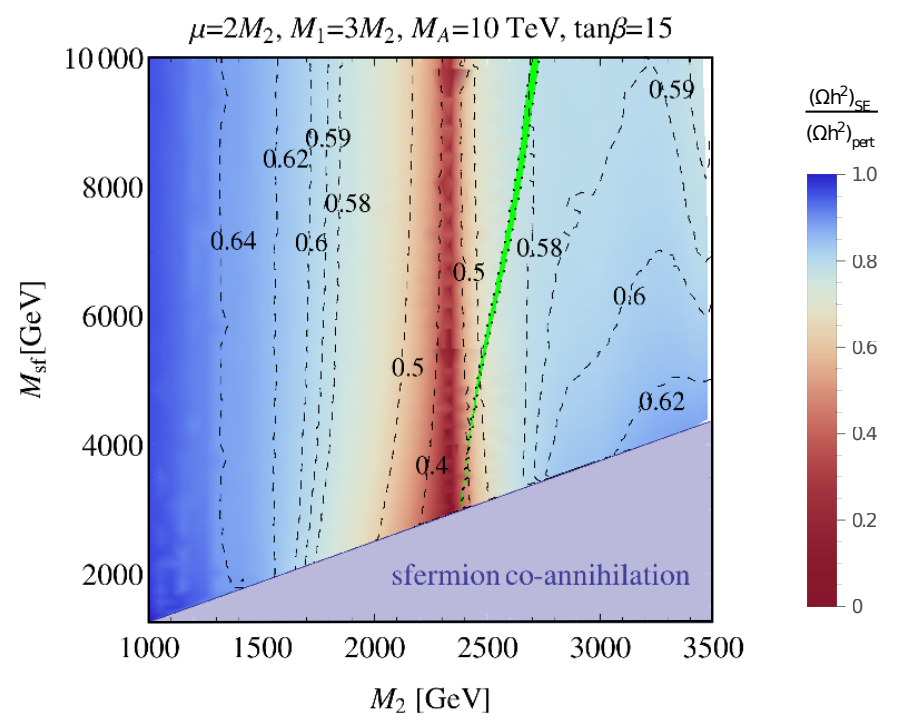

Figure 2. The ratio of the relic density including Sommerfeld enhancement to the perturbative result is shown via a density map as well as black dashed contours. The (green) band indicates the region within $2 \sigma$ of the observed dark matter abundance. Other parameters are as indicated, with $A_{i}=8 \mathrm{TeV}$ and $X_{t}$ is fixed by the measured Higgs mass. 
At the $\mathrm{TeV}$ scale the degeneracy between the charginos and neutralinos is more pronounced resulting in co-annihilation channels not being Boltzmann suppressed. Therefore the effective annihilation cross section is strongly affected by the co-annihilation channels e.g. from the processes $\tilde{\chi}_{1}^{+} \tilde{\chi}_{1}^{-} \rightarrow \bar{f} f, \tilde{\chi}_{1}^{0} \tilde{\chi}_{1}^{ \pm} \rightarrow \bar{f}^{\prime} f$. Due to interference between the sfermion t-channel and $W$ boson s-channel diagrams, the lower the sfermion masses, the smaller the contribution from these processes, leading to lower total annihilation cross section and higher thermal relic density. In other words, the contours of constant relic density move towards lower $m_{\mathrm{LSP}}$ values as the sfermion masses decrease. This is indeed what is observed in the left panel of figure 1, where the contours of constant perturbative relic density are plotted in the $M_{2}-M_{\mathrm{sf}}$ plane for the case of a wino-like LSP $\left(\mu=2 M_{2}\right.$, $\left.M_{1}=3 M_{2}\right)$. In particular note that, by varying the sfermion masses one can obtain the perturbative thermal relic density in agreement with the observed abundance over a large range $(\sim 800 \mathrm{GeV})$ of LSP masses. It is also worth pointing out that the fact that the contours become denser as $M_{2}$ increases is a simple result of the approximate quadratic dependence of the relic density on the wino mass.

The situation becomes more involved at the non-perturbative level, as shown in the right panel of figure 1. The main features that were previously discussed for the perturbative case are still present, but two important modifications arise. First, the contours are seen to be shifted towards larger values of $M_{2}$. This is simply the effect of the Sommerfeld enhancement on the annihilation cross section, such that one requires a wino mass of around $2.9 \mathrm{TeV}$ rather than $2.2 \mathrm{TeV}$ in order to obtain the correct thermal relic density in the decoupled sfermion case. The size of the shift however depends on the masses of the sfermions, in particular the lowest wino mass giving the correct relic density $\left.\Omega h^{2}\right|_{\exp }=0.1188 \pm 0.0010$ [60] without sfermion co-annihilations is around $2.3 \mathrm{TeV}$. This is related to the second effect, namely the resonance in the Sommerfeld enhancement, which is also responsible for lowering the constant relic density contours in the sfermion mass at $m_{\mathrm{LSP}}$ of around $2.3-2.4 \mathrm{TeV}$. The presence of the resonance is most clearly seen in figure 2 where the impact of the Sommerfeld effect on the relic density is shown. It can be seen that, as expected, the Sommerfeld effect gets stronger for larger values of $M_{2}$ until the resonance region is reached, and that in the resonance region the relic density can be suppressed by nearly an order of magnitude. What is worth stressing is that the Sommerfeld effect is also approximately independent of the value of the sfermion masses. This can be easily understood by noting that the largest impact of the Sommerfeld effect comes from its contribution on the $\tilde{\chi}_{1}^{0} \tilde{\chi}_{1}^{0}$ annihilation, which does not depend in any significant way on the nature of the sfermions. We also note that the Sommerfeld effect changes the relic density by almost a factor of two in the region where the observed relic density is attained (green bands in the figures), and by an even larger factor for smaller $M_{\mathrm{sf}}$, when the observed relic density is produced near the Sommerfeld resonance.

The generic behaviour of the results for the relic density as a function of sfermion masses shown and discussed above holds when one departs from wino-like neutralino as well, but with the details depending on the precise neutralino composition and the spectrum of the sfermions. The latter comes from the fact, that while the coupling of the sfermions with the wino is purely gauge, the one with the Higgsino is Yukawa-type, and therefore 
discriminates the three generations, as well as squarks from sleptons. The analysis of such scenarios, with Higgsino- and bino-like neutralinos, will be provided in the future.

\subsection{Higgsino admixture}

The Higgsino-wino mixing predominantly depends inversely on the difference between $\mu$ and $M_{2}$, as discussed in section 2.1. Increasing the Higgsino component of the predominantly wino-like LSP has several effects on the relic density: i) it modifies the LSP annihilation cross section due to different couplings of the wino and Higgsino components, ii) it changes the relevant number and weights of the co-annihilation channels and finally iii) it significantly alters the Sommerfeld effect. The first two effects are very well known, we therefore concentrate on the non-perturbative effects. We choose to parametrise the Higgsino admixture via the difference between the input parameters $\mu$ and $M_{2}$. For definiteness, we restrict ourselves to positive $\mu$ in the following analysis. In the $m_{\mathrm{LSP}}$ range considered, values of $\mu-M_{2} \gtrsim 500 \mathrm{GeV}$ lead to nearly decoupled Higgsinos, and the LSP is practically purely wino-like, while values of around $300-500 \mathrm{GeV}$ correspond to a Higgsino fraction of around a few $\%$, growing up to $50 \%$ for $\mu=M_{2}$.

The results of the analysis are displayed on figures 3 to 5 . In figure 3 the contours of constant relic density are shown in the $M_{2}$ vs. $\left(\mu-M_{2}\right)$ plane for the perturbative (left plot, blue lines) and Sommerfeld enhanced (right plot, red lines) cases. In the upper region of the plot the contour lines flatten as we recover the pure wino scenario, while in the lower region they tend to lower values of $m_{\mathrm{LSP}}$ because the large Higgsino fraction suppresses the annihilation cross section. Equivalently, for a fixed LSP mass, increasing the Higgsino admixture increases the relic density. At the perturbative level this is mainly a consequence of the lower value of the coupling to gauge bosons, while in the case of the Sommerfeld effect it also is a result of the larger mass splitting between the LSP and the lightest chargino. In figure 4 we show the ratio of the above plots in order to display the impact of the Sommerfeld enhancement over the $M_{2}$ vs. $\left(\mu-M_{2}\right)$ plane.

In figure 5 we show those contours giving the correct thermal relic density, for three different values of the sfermion mass parameter $M_{\mathrm{sf}}$, and show both the perturbative (left three lines, blue) and Sommerfeld-corrected (right three lines, red) results in one plot. The $M_{\mathrm{sf}}=12 \mathrm{TeV}$ lines correspond to the (green) bands in the previous figures 3 and 4 . We change here from displaying contours of constant relic density to the correct relic density in order to highlight the effect of the sfermion mass parameter. Note that, in agreement with what was discussed in the previous section, the lower the sfermion masses, the larger the relic density and hence the lower the LSP mass at correct relic density - both for the perturbative and non-perturbative results.

Comparing the perturbative result to the full one in figure 5, one observes the following:

i) the contours shift to higher masses, indicating the decrease of the relic density with respect to the perturbative result; in particular the mass of the lightest neutralino (when $\mu>M_{2}$ ) giving correct thermal relic density is around $1.7 \mathrm{TeV}$,

ii) for LSP masses in the range $2-3 \mathrm{TeV}$ the contours take a non-trivial form, which can be understood in terms of the resonance in the Sommerfeld enhancement. This 

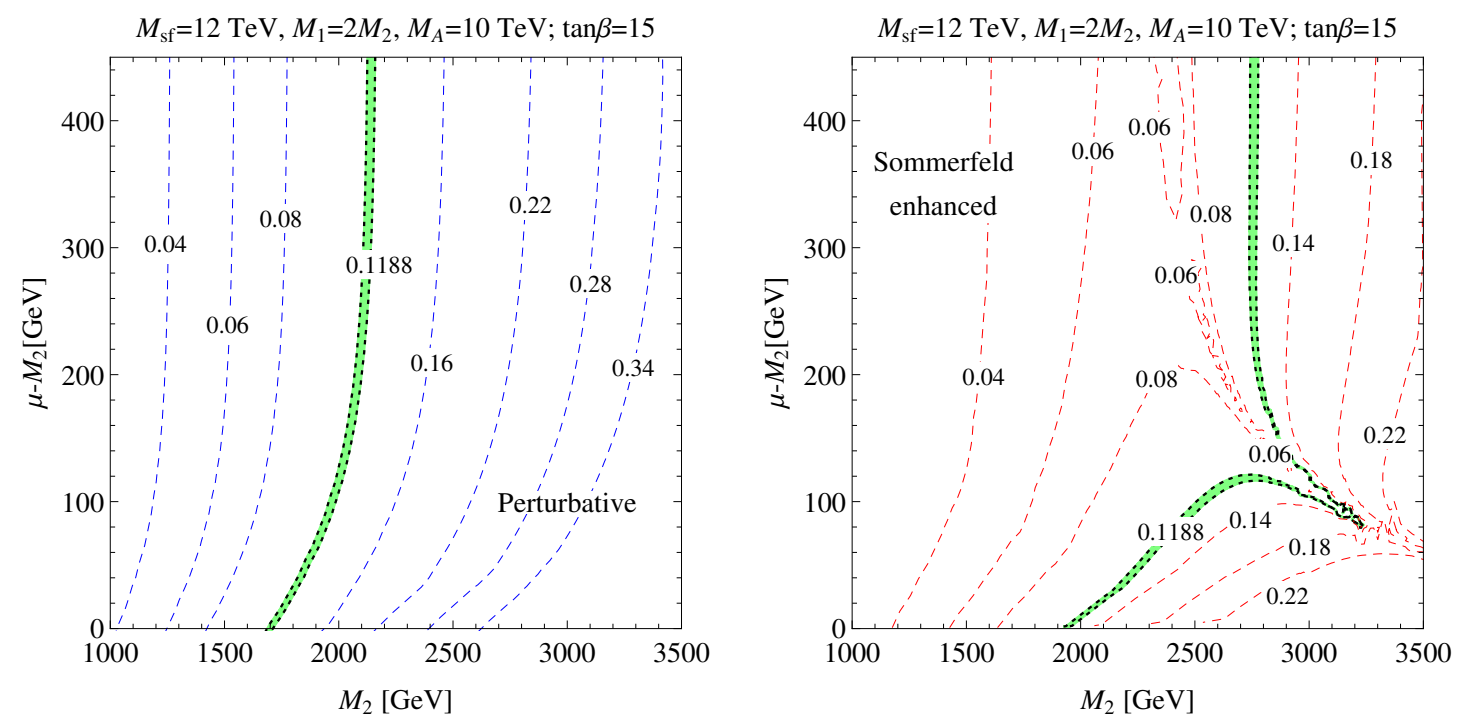

Figure 3. Contours of constant relic density are shown for the case of the perturbative (left) and Sommerfeld enhanced (right) calculation. The (green) bands indicate the region within $2 \sigma$ of the observed dark matter abundance. Other parameters are as indicated, with $A_{i}=8 \mathrm{TeV}$ and $X_{t}$ is fixed by the measured Higgs mass.

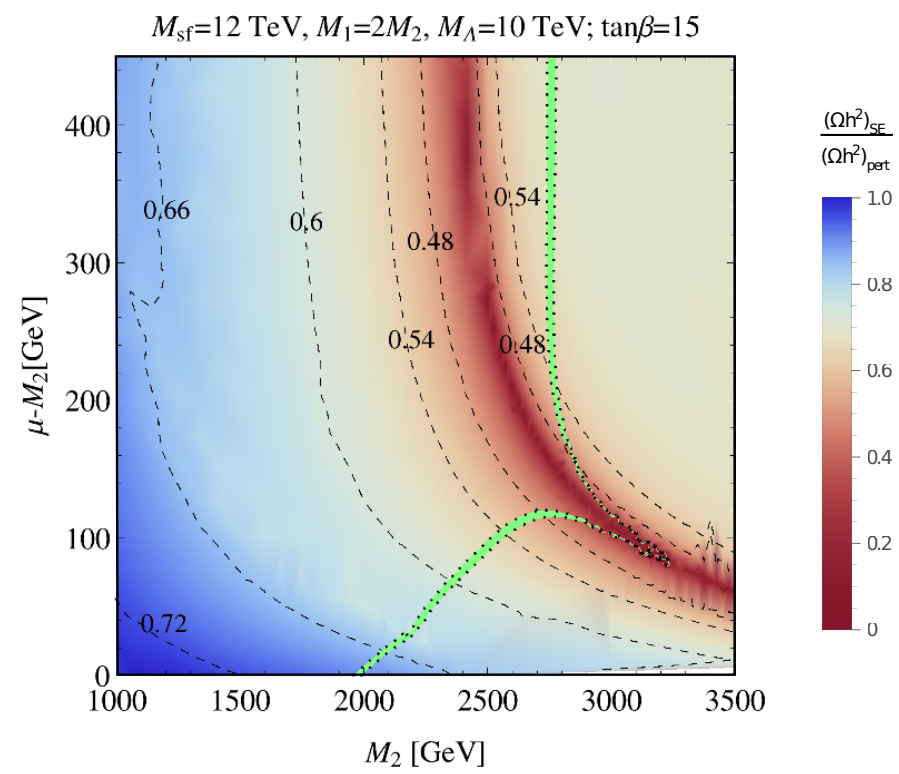

Figure 4. The impact of the Sommerfeld enhancement of the relic density shown as a density map as well as via the black dashed contours. The (green) band indicates the region within $2 \sigma$ of the observed dark matter abundance. Other parameters are as indicated, with $A_{i}=8 \mathrm{TeV}$ and $X_{t}$ is fixed by the measured Higgs mass. 


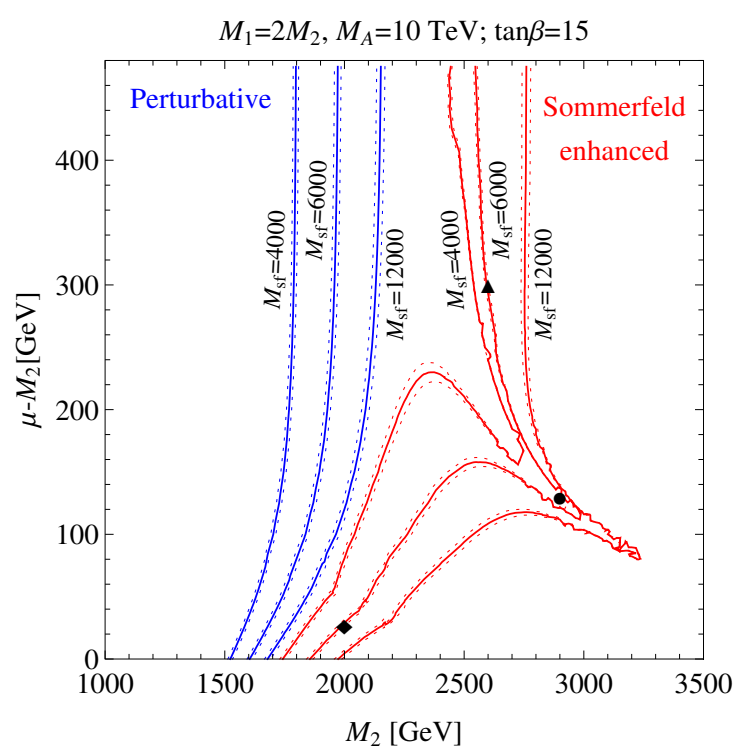

Figure 5. Contours providing the correct relic density are shown for the case of the perturbative (blue) and Sommerfeld enhanced (red) calculation for three different values of the common sfermion mass parameter. Other parameters are as indicated, with $A_{i}=8 \mathrm{TeV}$ and $X_{t}$ is fixed by the measured Higgs mass to a different value depending on $M_{\mathrm{sf}}$. The black markers denote the three points studied in section 3.5 .

resonance leads to much more efficient annihilation, strongly suppressing the relic density; it appears at different positions in $m_{\mathrm{LSP}}$ depending on the neutralino composition. In particular, on increasing the Higgsino fraction, the resonance occurs for heavier LSPs, which is mainly due to the increasing of the mass splitting between the lightest chargino and neutralino, the decreasing coupling, and the fact that the resonance depends on the splitting through (mass splitting) $/\left(m_{\mathrm{LSP}} \alpha_{2}^{2}\right)$.

The position of the peak of the resonance was clearly visible in figure 4 . It is therefore evident that in figure 5 the contours of the correct relic density cluster around this peak for higher values of $M_{2}$ and lower values of $\mu-M_{2}$. This is easily understood when one recalls that in this region the neutralino at a perturbative level has a thermal abundance larger than that observed by a factor of a few. The proximity to the resonance enhances the cross section, reducing the relic density to agree with the measured value. In particular, it follows that the largest value of $M_{2}$ giving the correct thermal relic density is close to $3.3 \mathrm{TeV}$, approximately $20 \%$ higher than that for the pure-wino scenario.

Note also that, in contrast to the pure-wino scenario with decoupled sfermions, a region of parameter space exists where the thermal relic density is obtained in very close vicinity to the resonance, leading to strong bounds on such scenarios coming from dark matter indirect searches. Previously only limiting wino and Higgsino cases have been studied from this perspective with the inclusion of the Sommerfeld effect $[8,61,62]$, and even slightly mixed scenarios remain unexplored. ${ }^{9}$ It also follows that some regions of the pMSSM

\footnotetext{
${ }^{9}$ The only related works available in the literature [63-65] are considering the Sommerfeld effect in an
} 
parameter space can be effectively constrained by non-observation of any dark matter signal in cosmic or $\gamma$-rays. The precise analysis of such phenomenologically interesting regions will be presented in upcoming work [66].

\subsection{Effect of the heavy Higgs bosons}

In the MSSM, the only particles beyond the SM having positive R-parity are the additional Higgs bosons. These can therefore act as an s-channel mediator and, if light enough, as end-products of the (co-)annihilation. As the effect of these Higgs bosons is greatest when the Higgsino mass parameter is close to $M_{2}$, we discuss this first before moving on to the wino-bino mixed case. The additional Higgs bosons can affect the relic density in the following two ways:

- by contributing to the (co-)annihilation rate via s-channel diagrams, particularly if $M_{A}$ lies in the vicinity of $2 m_{\mathrm{LSP}}$, thereby typically reducing the relic density

- by providing additional final states with one heavy Higgs plus one gauge or light Higgs boson, or with two heavy Higgs bosons, if the combined mass of the final state lies below $2 m_{\mathrm{LSP}}$, which leads to a reduction in the relic density.

The former is only relevant when the coupling of the annihilating particles to the Higgs bosons is non-negligible. This requires one of the two annihilating neutralinos or charginos to contain a considerable gaugino component and the other a considerable Higgsino component. For $\tilde{\chi}_{0}$ annihilation this implies that the LSP is mixed. We remind the reader that we do not consider the resonant annihilation region when $M_{A}$ is inside the interval 1.7-2.3 $m_{\mathrm{LSP}}$ as explained in section 2.2. As for the latter point, the heavy Higgs and gauge boson final state is obtained via a s-channel gauge boson, or a t-channel neutralino or chargino. This is again more relevant when the LSP contains some Higgsino admixture, as this also allows the coupling of neutralinos to $Z$ bosons. However, in contrast to the case of the heavy Higgs boson in the s-channel, this contribution does not vanish when the Higgsino decouples, as a coannihilating chargino and neutralino can annihilate into a heavy Higgs and gauge boson via a s-channel $W$ boson even in the pure-wino limit.

We explore these issues in figure 6 where we show contours of constant relic density in the $M_{2}$ vs. $M_{A}$ plane both at the perturbative level (left) and on taking account of the Sommerfeld effect (right). The region corresponding to the measured relic density is shown by the green band. As the s-channel resonance cannot be accurately calculated in our framework we do not provide results near $M_{A}=2 M_{2}$. It is seen that for the perturbative case above the excluded region the lines are approximately vertical, just bending slightly towards higher values of $M_{2}$ on approaching this region. Below the excluded area we find that there is a slight shift to the right as the heavy Higgs bosons are accessible in the final state. The difference in $M_{2}$ giving the correct relic density is approximately $150 \mathrm{GeV}$ when $M_{A}$ changes from $10 \mathrm{TeV}$ to $500 \mathrm{GeV}$. For the Sommerfeld-enhanced case the result is qualitatively similar, however the difference in $M_{2}$ giving the correct relic density is around $250 \mathrm{GeV}$ for the same change of $M_{A}$.

approximate way and/or without inclusion of recent developments [12-14]. 

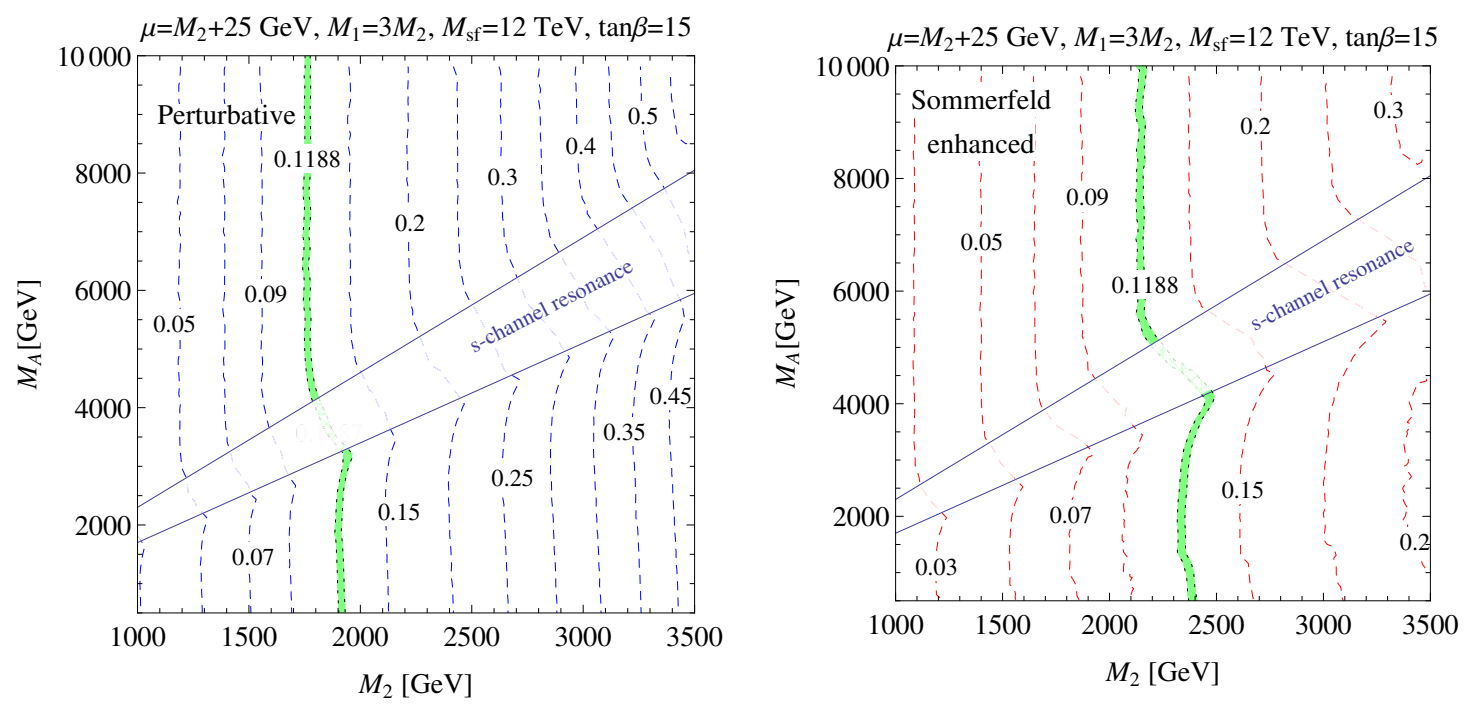

Figure 6. Contours of constant relic density are shown for the case of the perturbative (left) and Sommerfeld enhanced (right) calculation. The (green) bands indicate the region within $2 \sigma$ of the observed dark matter abundance. Other parameters are as indicated, with $A_{i}=8 \mathrm{TeV}$ and $X_{t}$ is fixed by the measured Higgs mass.

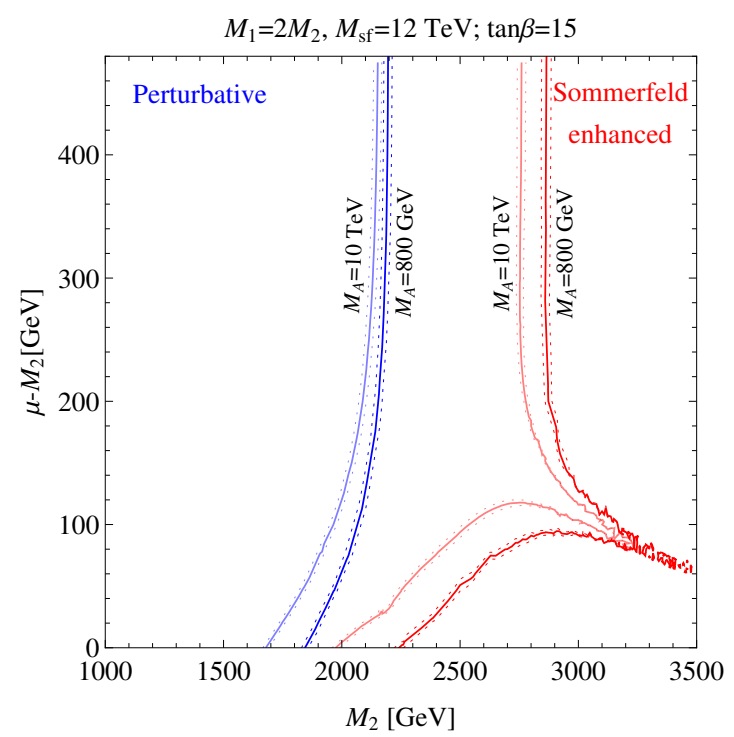

Figure 7. Contours providing the correct relic density are shown for the case of the perturbative (blue) and Sommerfeld enhanced (red) calculation for three different values of the heavy Higgs mass $M_{A}$. Other parameters are as indicated, with $A_{i}=8 \mathrm{TeV}$ and $X_{t}$ is fixed by the measured Higgs mass. 
In figure 7 we further investigate the effect of the heavy Higgs bosons on the contours showing the correct relic density in the $M_{2}$ vs. $\mu-M_{2}$ plane. The blue lines show the perturbative result while the red lines include the Sommerfeld enhancement. We see that on decreasing $M_{A}$ from $10 \mathrm{TeV}$ to $800 \mathrm{GeV}$, the shift in the value of $M_{2}$ giving the correct density is indeed dependent on the proximity of $\mu$ to $M_{2}$, increasing from 50 to $150 \mathrm{GeV}$ in the perturbative case and 100 to $250 \mathrm{GeV}$ in the Sommerfeld-enhanced case. As mentioned earlier, an increased Higgsino admixture allows a stronger coupling to the Higgs and $Z$ bosons in the s-channel (where $Z$ bosons can give rise to heavy Higgs bosons in the final state), increasing the effect of the heavy Higgs boson. Nevertheless when the Higgsino is decoupled a dependence on $M_{A}$ persists; for the $M_{A}=800 \mathrm{GeV}$ contours coannihilation via a $W$ boson to a final state containing a heavy Higgs boson and a gauge boson is allowed but not for the $M_{A}=10 \mathrm{TeV}$ contours.

\subsection{Bino admixture}

The bino only mixes with the wino via the off-diagonal terms in the Higgsino block of the neutralino mass matrix. It follows that the mixing is weak, depending of course on the Higgsino parameter $\mu$, and is further sensitive to $\tan \beta$ and the sign of $M_{1}$ and $\mu$ as seen in eq. (2.5). In order that the wino-like neutralino contains a substantial bino component, either $\mu$ should be of the same order as $M_{1}$ and $M_{2}$ or the $M_{1}$ and $M_{2}$ parameters should be highly degenerate. For example, when $\delta M_{1}=M_{2}-M_{1}=10 \mathrm{GeV}, \mu=2 M_{2}$ and $\tan \beta=15$ the mixing is about $1 \%$, decreasing to $0.1 \%$ when $\delta M_{1}=100 \mathrm{GeV}$. Such situations may arise and are worth studying as the resulting features are of phenomenological interest. In this section we focus on the second case $M_{1} \sim M_{2} \ll \mu$, since the first $\left(M_{1} \sim M_{2} \sim \mu\right)$ falls into the category of a mixed wino-Higgsino state and shares the gross features with the case of a decoupled bino analysed in the previous section. We further assume $M_{1}>0$, since $M_{1}<0$ entails an essentially decoupled bino.

When $M_{1}$ is close to $M_{2}$, the perturbative relic density is affected both by the modification of the LSP annihilation cross section due to the change in composition and by the co-annihilation with the bino-like NLSP, with mass close to $M_{1}$. On top of that the Sommerfeld effect is modified, analogously to the Higgsino-wino mixed scenario, by the weakened coupling and larger mass splitting between $\tilde{\chi}_{1}^{0}$ and $\tilde{\chi}_{1}^{ \pm}$as given in eqs. (2.6), (2.7). Qualitatively the behaviour observed on increasing the bino component is largely the same as in the Higgsino case, with an important quantitative difference: a larger sensitivity of the results to the remaining parameters.

Contours of constant relic density in the $M_{2}$ vs. $\left(M_{1}-M_{2}\right)$ plane for $M_{1}>0$, and both the perturbative and Sommerfeld enhanced case are displayed in figure 8. Note that the logarithmic scale for the vertical axis, chosen due to the weak mixing between the bino and the wino, changes the appearance of the resonance with respect to the Higgsino case. As one increases the bino component the mass of the LSP resulting in the correct relic density is approximately $1500 \mathrm{GeV}$ rather than $1800 \mathrm{GeV}$ for the perturbative case. This changes rather dramatically when the Sommerfeld enhancement is taken into account, notably for strong mixing, i.e. $M_{1}-M_{2} \lesssim 10 \mathrm{GeV}$, there are three values of $M_{2}$ which give the correct 

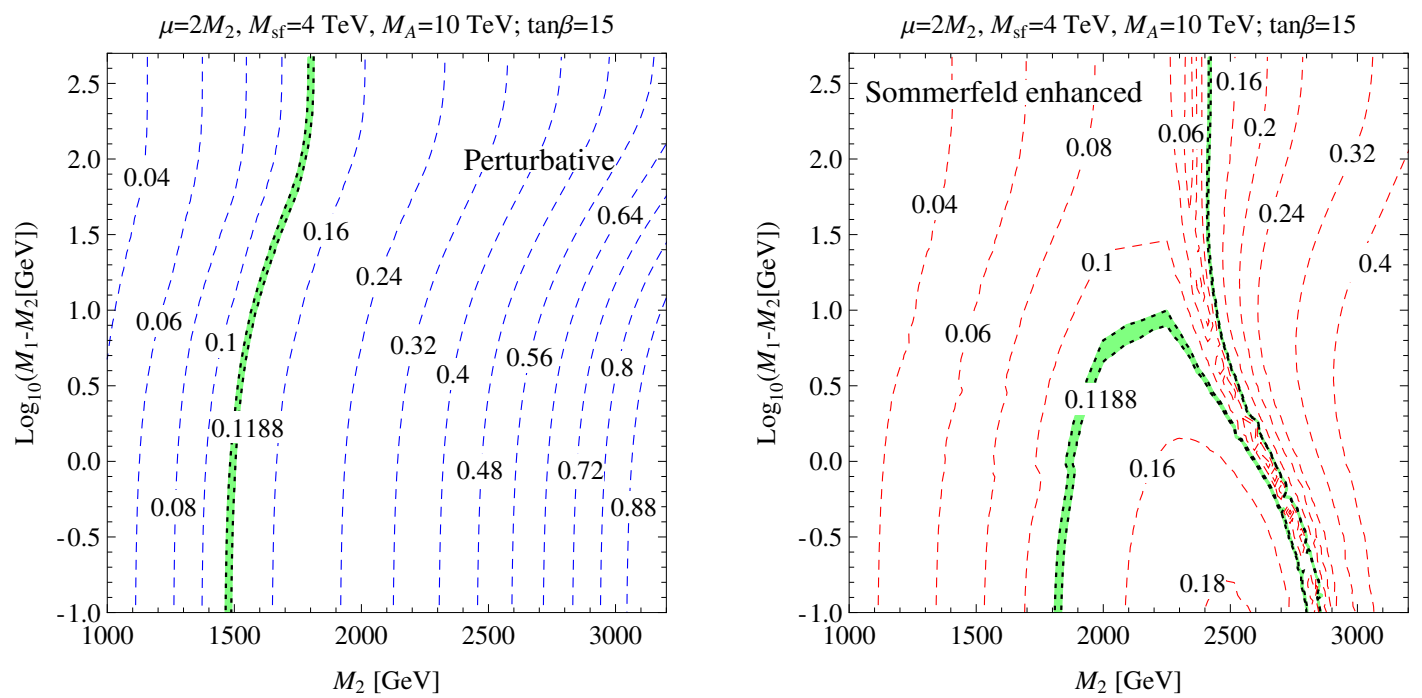

Figure 8. Contours of constant relic density are shown for the case of the perturbative (left) and Sommerfeld enhanced (right) calculation. The (green) bands indicate the region within $2 \sigma$ of the observed dark matter abundance. Other parameters are as indicated, with $A_{i}=8 \mathrm{TeV}$ and $X_{t}$ is fixed by the measured Higgs mass.

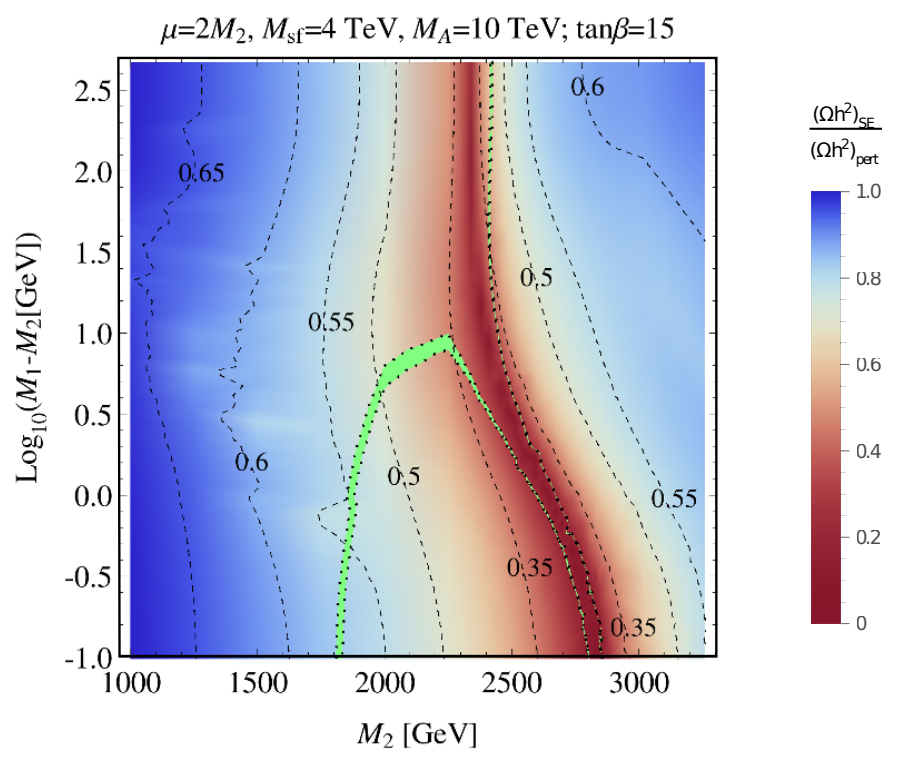

Figure 9. The impact of the Sommerfeld enhancement of the relic density shown as a density map as well as via the black dashed contours. The (green) band indicates the region within $2 \sigma$ of the observed dark matter abundance. Other parameters are as indicated, with $A_{i}=8 \mathrm{TeV}$ and $X_{t}$ is fixed by the measured Higgs mass. 


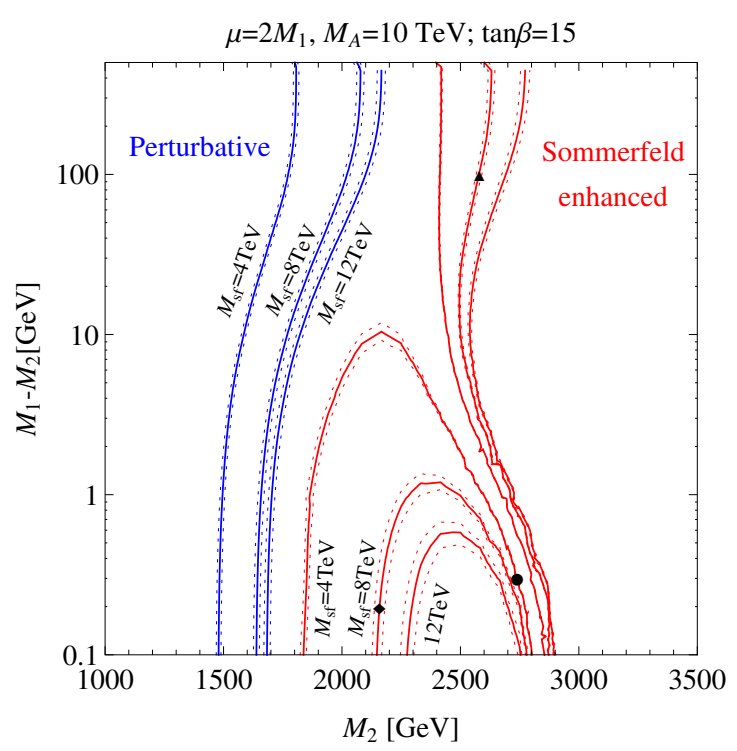

Figure 10. Contours of correct relic density: perturbative (blue) and Sommerfeld enhanced (red) are shown for 3 different values of the sfermion mass parameter. Other parameters are as indicated, with $A_{i}=8 \mathrm{TeV}$ and $X_{t}$ is fixed by the measured Higgs mass. The black markers denote the three points studied in section 3.5 .

relic density. One can interpret the larger two of these values as a result of the resonance in the Sommerfeld enhancement.

In figure 9 we study the ratio between the relic densities shown in figure 8 , in terms of a density plot with contour lines overlaid. The correct relic density for the full calculation including the Sommerfeld enhancement is highlighted by the (green) band. We observe the maximal effect of the Sommerfeld enhancement is in fact in the region where the relic density agrees with observation, in particular when the difference between $M_{1}$ and $M_{2}$ is below approximately $10 \mathrm{GeV}$, the Sommerfeld enhanced relic density in agreement with that observed is three times smaller than the perturbative result at the same parameter values. Note that over the entire region covered by the plot the effect of the Sommerfeld enhancement is greater than $30 \%$.

In figure 10 contours with the correct relic abundance for three choices of the sfermion mass parameter are shown. The results again resemble the Higgsino admixture case, up to differences already commented on. Note that in the region around the resonance, the effect of the sfermion masses is less pronounced than elsewhere. One observes that the lowest mixed wino-bino neutralino mass giving the observed relic density is around $1.8 \mathrm{TeV}$ for $M_{\mathrm{sf}}=4 \mathrm{TeV}$, marginally higher than the wino-Higgsino case. The highest value is $2.9 \mathrm{TeV}$ (for $M_{\mathrm{sf}}=12 \mathrm{TeV}$ ) compared to $3.3 \mathrm{TeV}$ in figure 5 . However, as can be seen in figure 11, the highest value of $M_{2}$ resulting in the correct relic density is strongly dependent on the value of the $\mu$ parameter, as this mediates the mixing. This dependence is demonstrated via contours for five different choices of $\mu$. The contour for $\mu=1.1 M_{1}$ bears a closer resemblance to the wino-Higgsino case, as suggested earlier. Note that as $\mu$ decreases, 


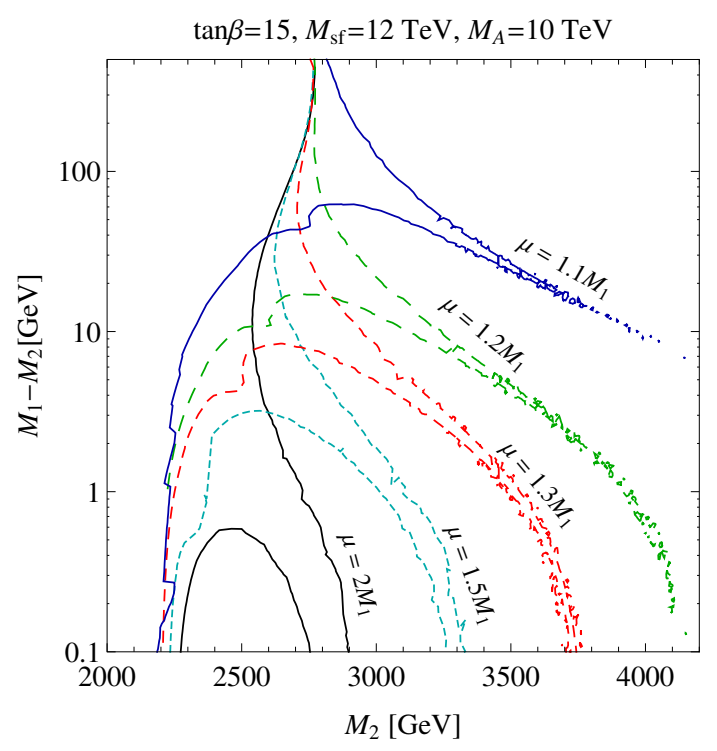

Figure 11. Contours of correct relic density for different values of $\mu$. Other parameters are as indicated, with $A_{i}=8 \mathrm{TeV}$ and $X_{t}$ is fixed by the measured Higgs mass.

the lightest chargino-neutralino mass splitting for a given point in the plane increases, ${ }^{10}$ resulting in the resonance moving to higher values of $m_{\mathrm{LSP}}$. Due to the presence of the resonance, it appears that by making an appropriate choice in $\mu$ and $M_{1}$ the entire region could be covered, at least for values of $M_{2}$ from 2100 to $4200 \mathrm{GeV}$ if not even higher. All these points would be on or around the resonance, having implications for Indirect Detection. Moreover, interestingly adding a bino component to the LSP can extend the possible neutralino masses giving observed dark matter abundance up to and even beyond $4.1 \mathrm{TeV}$.

\subsection{Residual dependence on other parameters}

In the previous sections 3.1 to 3.4 certain parameters were fixed in order to obtain a clearer understanding of the dependence of the results on the central parameters $M_{1}, M_{2}, \mu, M_{\mathrm{sf}}$, as well as on $M_{A}$. However, it is important to confirm whether these are indeed the most relevant, and to investigate the effect of the other parameters, e.g. $\tan \beta$, which was so far neglected.

One case in which additional parameters may play a significant role is when the lightest neutralino is a wino-bino mixture as in section 3.4. This is because, as seen in eqs. (2.5) to (2.7), the mixing of the wino with the bino, and the splitting between the lightest neutralino and chargino is sensitive to $|\mu|$, the sign of $\mu$ and $\tan \beta$. The dependence on $\mu$ was already examined in section 3.4, and the results can be found in figure 11. Here we further consider the effect of the sign of $\mu$ and the choice of $\tan \beta$. Our results can be found in figure 12. The benchmark choice for the results presented in previous subsections was $\tan \beta=15$ and $\mu>0$; in addition here we consider $\mu>0$ with $\tan \beta=5,30$ and $\mu<0$ with $\tan \beta=15$. Large deviations from the benchmark scenario are seen in the resonant region.

\footnotetext{
${ }^{10}$ See figure 15 in the appendix.
} 


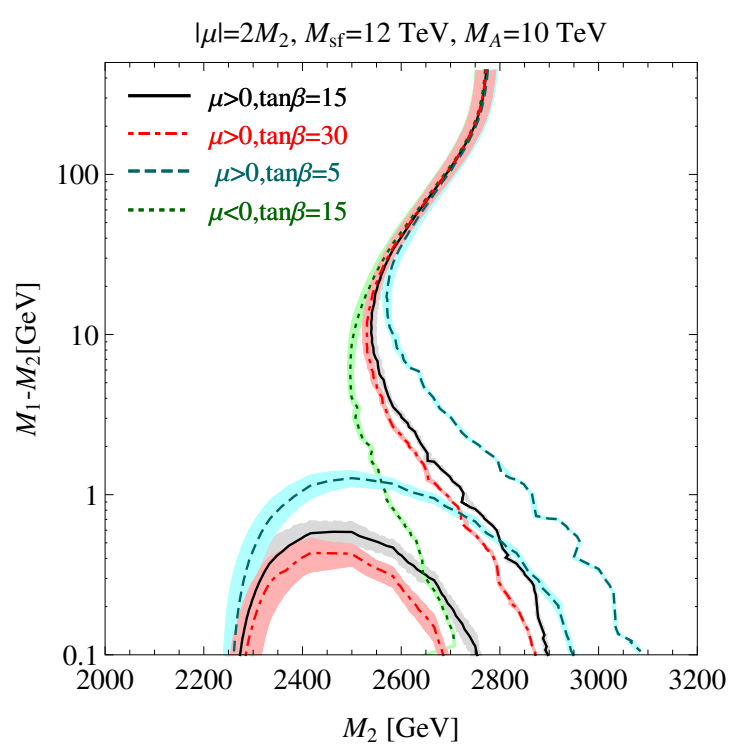

Figure 12. The contours of correct relic density for different choices of $\tan \beta$ and the sign of $\mu$. Other parameters are as indicated, with $A_{i}=8 \mathrm{TeV}$ and $X_{t}$ is fixed by the measured Higgs mass.

This can be understood by examining the expressions for the mass splitting between the lightest neutralino and chargino in eqs. (2.6) and (2.7). The splitting is seen to increase when $\tan \beta$ decreases, and also when $\mu>0$ compared to $\mu<0$, resulting in the position of the resonance moving towards higher values of $m_{\mathrm{LSP}}$, i.e. the correct relic density is observed for higher $M_{2}$.

We further examine the sensitivity to the remaining parameters for both the cases of mixed wino-Higgsino and wino-bino LSPs in figure 13. To this end we show histograms of the percentage of points in bins of $\Omega h^{2} /\left\langle\Omega h^{2}\right\rangle$ where $\left\langle\Omega h^{2}\right\rangle$ is the mean value of the relic density, both for the perturbative and the full calculation. We choose six wino-like points, three of which contain bino admixtures and three Higgsino admixtures of varying degree. The points in the $\left(M_{2}, \mu-M_{2}\right)$ and $\left(M_{2}, M_{1}-M_{2}\right)$ planes corresponding to the left and right hand plots from top to bottom are marked (up to signs in $\mu, M_{1}$ ) in figures 5 and 10 by the triangle, circle and diamond, respectively, i.e. the Higgsino or bino component increases from top to bottom panels. For each of these points we fix the values of the central parameters as indicated in figure 13 and compute the relic density for 1000 different realisations of the remaining parameters $M_{A}$ (for the case of wino-bino mixing), $\tan \beta$ and $A_{f}$ within the ranges given in table 1, with $M_{3}$ fixed to $3 M_{2}$, assuming a uniform distribution before the constraints are imposed.

The perturbative results are shown by the dark-grey/blue histograms. We see that in all cases the distribution is strongly peaked near the central value with the variation of order of at most a few per cent, and that the distribution widens with the departure from the pure-wino limit. The situation changes when considering the full result (light-grey/red histograms), as all the distributions become broader and asymmetric. The position of the resonance in the Sommerfeld effect is greatly sensitive to values of the neutralino mass, 

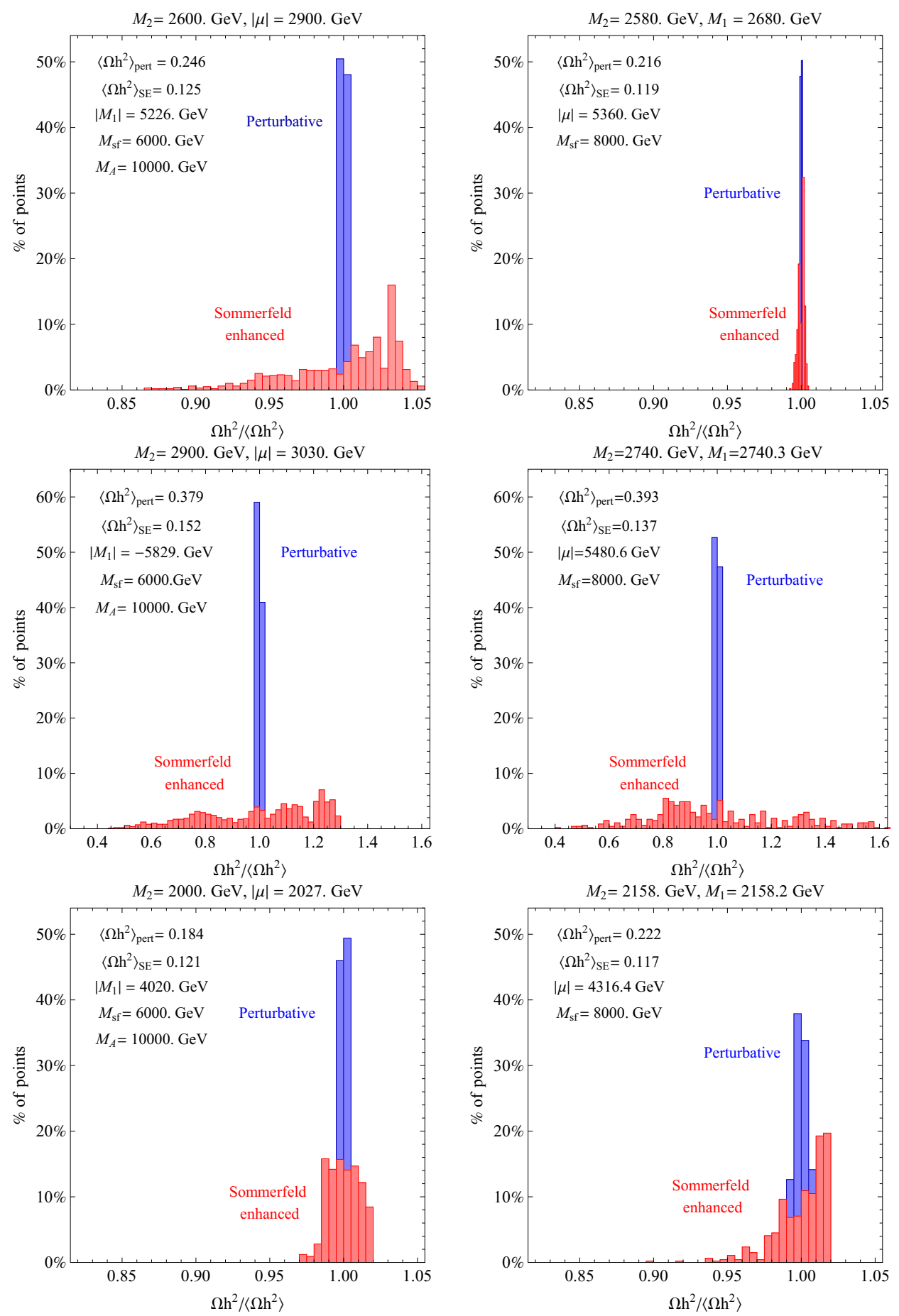

Figure 13. Histograms showing the impact of the remaining parameters on the relic density for wino-like LSPs with a varying Higgsino (left) and bino (right) admixtures. The relic density is normalised to the mean for each case respectively. 
couplings and the chargino. Therefore, slight changes in these values caused by different choice of remaining less relevant MSSM parameters, especially close to the resonance, can lead to observable differences in the relic density. Indeed, the broadening of the full result with respect to the perturbative one is strongest in the middle panel (due to the vicinity of the circle benchmark point to the resonance) and in the upper left-hand plot being not far from the resonance as well. The asymmetry in the distributions is caused by the fact that deviations around central parameters may go towards or away from the resonance, leading to larger or smaller Sommerfeld effects respectively. The bottom line of this analysis is that away from the resonance the residual MSSM parameters have a very mild impact, justifying our choice of central parameters, while in the vicinity of the resonance regions the variation is very significant.

To study the dependence on the residual parameters even further, we have generated a large number of points (50000 and 90000 for the Higgsino and bino case, respectively), where we considered the wino mass in the range $M_{2} \in\{1,3.5\} \mathrm{TeV}$ and (different from table 1 and the analyses in the previous sections) fixed the gluino mass parameter via $M_{3}=2 M_{2}$. The sfermion masses were fixed to the values given below, but we varied all other parameters in the following ranges:

$$
\frac{X_{t}}{M_{\mathrm{sf}}} \in\{0.5,3\}, A_{f} \in\{0,8\} \mathrm{TeV}, M_{A} \in\{1,10\} \mathrm{TeV}, \tan \beta \in\{5,30\},
$$

where $f$ in $A_{f}$ includes all fermions except the top. In addition, for the Higgsino case we chose:

$$
M_{\mathrm{sf}}=6 \mathrm{TeV},\left|M_{1}\right|=2.01 M_{2}, M_{2} \in\{1,3.5\} \mathrm{TeV}, \mu \in\left\{M_{2}, M_{2}+0.5 \mathrm{TeV}\right\},
$$

and for the bino case:

$$
M_{\mathrm{sf}}=8 \mathrm{TeV}, M_{1} \in\left\{M_{2}, M_{2}+0.1 \mathrm{TeV}\right\},|\mu|=2 M_{1} .
$$

From the generated points, we selected those where either the perturbative or the Sommerfeld enhanced relic density was found to be between 0.1168 and 0.1208 , i.e. within two sigma of the central value. In figure 14 we overlay these points on the relevant plots shown earlier, figures 5 and 10 for the Higgsino (upper plot) and bino (lower plot) case, respectively.

We observe that those points for which the perturbative relic density lies within $2 \sigma$ of the central value are located very close to the respective sfermion mass contours, the spread of the points being comparable to twice the width of the $1 \sigma$ contours. We conclude that the dependence on the residual parameters is very mild in this case. As could be expected, when including the Sommerfeld enhancement, the residual parameters can have a larger effect, especially close to the resonance. In order to investigate this effect further, for the wino-Higgsino case we have divided these points according to whether $2.3 m_{\mathrm{LSP}}<M_{A}<$ $2.5 m_{\mathrm{LSP}}, M_{A}>2.5 m_{\mathrm{LSP}}$ and $M_{1}>0$, or $M_{A}>2.5 m_{\mathrm{LSP}}$ and $M_{1}<0$. These are indicated in figure 14 by the cross, filled circle and open circle respectively. The former division is made in order to isolate those points in proximity to the heavy Higgs funnel region and the latter due to the effect of the sign on $M_{1}$ on the lightest neutralino-chargino mass splitting. 

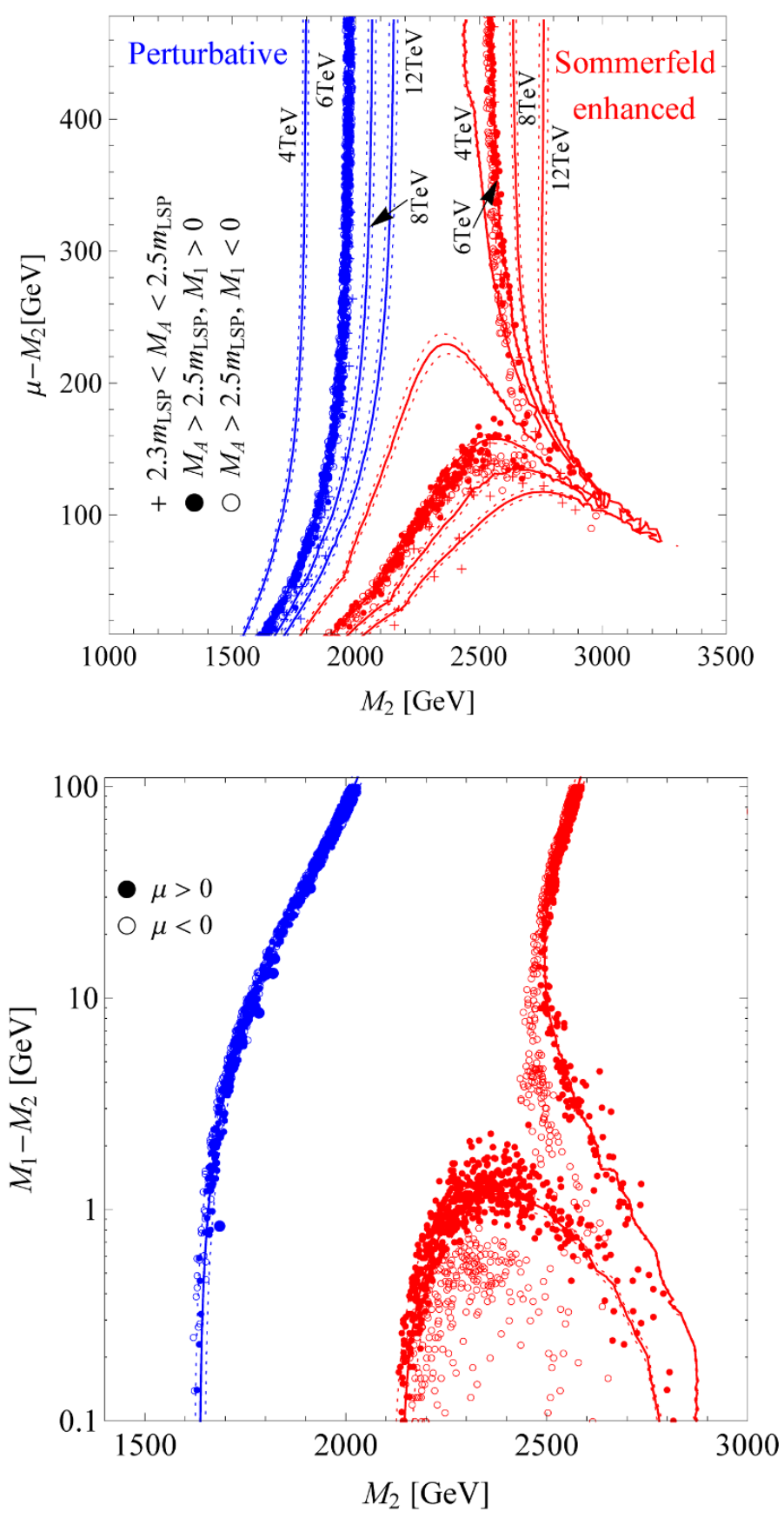

Figure 14. Plots showing points satisfying the relic density constraint obtained on varying the parameters $\tan \beta, M_{A}$ and $A_{f}$ for wino-like LSPs with varying Higgsino (upper) and bino (lower) admixtures. The points are overlaid on contours for fixed values of these parameters and $M_{\mathrm{sf}}$ as indicated. 
We do not plot the points with $M_{A}<1.7 m_{\mathrm{LSP}}$ in this case. For the wino-bino case we separated the points according to whether $\mu>0$ or $\mu<0$, indicated in figure 14 by the filled circle and open circle respectively, as the sign of $\mu$ also plays a role in the size of the lightest neutralino-chargino mass splitting.

The effect of the residual parameters is sub-dominant with respect to e.g. that of the sfermion masses, but both the sign of $\mu$ and $M_{1}$ are seen to play a role in the resonance region for the wino-bino and wino-Higgsino cases, respectively. This can be understood in terms of the expressions for the mass difference between the lightest chargino and neutralino in eqs. (2.3), (2.4) and (2.6), (2.7) to which the resonance is sensitive. As the splitting increases the position of the resonance moves towards higher values of $m_{\mathrm{LSP}}$. Whether the heavy Higgs is below, above, or, in particular, close to the excluded window also has a noticeable effect for the case of wino-Higgsino mixing, and this extends beyond the resonance region and holds for the perturbative case as well. This is because for states with larger mixing the coupling to the heavy Higgs is enhanced, and therefore when $M_{A}$ decreases the s-channel annihilation cross section increases, and one has to go to higher values of $M_{2}$ to obtain the correct relic density. This is not relevant for the wino-bino case, where the dependence on the value of $M_{A}$ is negligible.

To summarise, we find that the assumption that our results of the previous sections were more or less independent of certain parameters was largely justified. Only for the wino-Higgsino case there is some dependence on the value of $M_{A}$, and in the resonance region the sensitivity to these parameters is somewhat enhanced, particularly to the values of $\tan \beta$ and the sign of $\mu$ for the case of wino-bino mixing and $M_{1}$ for the case of winoHiggsino mixing.

\section{Summary}

We have studied the Sommerfeld effect on the relic density of neutralino dark matter beyond the pure-wino limit. This involved a scan of parameter space for three scenarios where the lightest neutralino contained a large wino component: one with non-decoupled sfermions, and the remaining with either a Higgsino or bino admixture. We aimed to determine how in these scenarios (a) the mass of the LSP where the relic density constraint is satisfied and (b) the size of the Sommerfeld enhancement is altered in comparison to the pure-wino case.

The calculation of the Sommerfeld enhancement for the scenarios in question required a consistent treatment of mixed neutralinos including multiple co-annihilation channels and off-diagonal contributions as well as of $\mathcal{O}\left(v^{2}\right)$ contributions. As the Sommerfeld effect, in particular the position of the resonance, depends strongly on the mass splittings between the neutralinos and charginos, we used a dedicated on-shell renormalisation scheme scheme for one-loop masses. As the relic density depends strongly on the precise value of the gauge coupling, we adopted running couplings. Further we have argued that the size of thermal corrections is sufficiently below the uncertainty of our calculation, i.e. the percent level, that they can be neglected. Finally, all points in MSSM parameter space considered were checked for consistency with current experimental measurements. Our calculation was 
carried out by a code which will be made available to the public: this will be presented in more detail in a separate publication.

Due to t-channel interference, we found that when the sfermions are non-decoupled they reduce the annihilation cross section such that the relic density constraint is satisfied at lower values of $m_{\mathrm{LSP}}$, from $2.9 \mathrm{TeV}$ for decoupled sfermions down to $2.4 \mathrm{TeV}$. For the mixed neutralino scenarios we found a much larger dependence than expected on those parameters affecting the mass splitting between the lightest chargino and neutralino. This was particularly evident in the mixed bino-wino region, where the position of the resonance was seen to be sensitive to $\mu, \tan \beta$ and the sign of $\mu$. As the splitting increases we observed that the resonance lies at higher values of $m_{\mathrm{LSP}}$. This led to a large range of neutralino masses from 1.8 to beyond $4 \mathrm{TeV}$ satisfying the relic density constraint. For the Higgsinowino mixed region the position of the resonance depends primarily on $\mu-M_{2}$, but whether the mass of the heavy Higgs boson lies below or above $2 m_{\text {LSP }}$ also plays a significant role. Here we found values of $m_{\mathrm{LSP}}$ ranging from 1.7 to $3.3 \mathrm{TeV}$.

This is the first time that the sensitivity of the Sommerfeld enhancement to MSSM parameters for mixed neutralinos has been studied systematically and to such accuracy, and the large range of possible $m_{\mathrm{LSP}}$ masses providing the correct relic density was previously unknown. In most of the cases the Sommerfeld effect changes the relic density by a factor of two or even higher relative to the tree-level computation. This underscores the fact that the relic density of $\mathrm{TeV}$ scale MSSM dark matter can usually not be predicted correctly without accounting for this effect.

In light of these results a re-investigation of the bounds on the Sommerfeld enhanced scenarios coming from Indirect Detection experiments is imperative. It is likely that so far unexplored regions exist, sufficiently far away from the resonance that they are not excluded, but with Sommerfeld enhanced annihilation cross sections which could be probed by upcoming experiments, e.g. the Cherenkov Telescope Array (CTA). This will be the subject of a dedicated study in the near future.

\section{Acknowledgments}

This work is supported in part by the Gottfried Wilhelm Leibniz programme of the Deutsche Forschungsgemeinschaft (DFG) and the Excellence Cluster "Origin and Structure of the Universe" at Technische Universität München. We further gratefully acknowledge that part of this work was performed using the facilities of the Computational Center for Particle and Astrophysics (C2PAP) of the Excellence Cluster.

\section{A Thermal effects}

Freeze-out of dark matter begins when the Universe has cooled to the temperature $T_{f} \simeq m_{\chi} / 20$. For the dark matter masses considered in this paper $T_{f}$ is in the range $50 \ldots 200 \mathrm{GeV}$, which includes the temperature $T_{c} \approx 165 \mathrm{GeV}$ of the electroweak phase transition. Above the critical temperature $T_{c}$, the Lagrangian mass of the electroweak gauge bosons vanishes and so does the neutralino-chargino mass splitting in the wino-like region. 
Furthermore, large thermal masses and mass splittings may be generated. While thermal effects on the short-distance annihilation process are small [67], the gauge-boson mass determines the range of the potential, which is an important quantity for the Sommerfeld effect. Furthermore, the mass splitting of the lightest chargino and neutralino influences the location of the Sommerfeld resonance. In the following, we investigate the thermal modification of the gauge boson mass and neutralino-chargino mass splitting through a combination of estimates, analytical calculations and numerical checks.

The relevant temperature range for this investigation is limited from above by $T \simeq$ $m_{\chi} / 20$, when freeze-out begins, which allows us to treat $T / m_{\chi}$ as small. The temperature of the Universe together with the Boltzmann distribution sets the characteristic scale of the three-momentum of the scattering dark matter particles to $|\vec{p}| \sim\left(m_{\chi} T\right)^{1 / 2}$. The Sommerfeld effect is caused by ladder diagrams with loop momentum $k$ satisfying $k^{0} \ll|\vec{k}| \ll m_{\chi}$ where $k^{0}$ is determined by the pole of the dark matter particle propagator. The characteristic scale of $\vec{k}$ is also $\left(m_{\chi} T\right)^{1 / 2}$ until $m_{\chi} T \sim m_{W}^{2}$, where $m_{W}$ is the mass of the exchanged electroweak gauge boson (zero for the photon), at which point the Sommerfeld enhancement saturates. When the Universe cools below $T_{s} \simeq m_{W}^{2} / m_{\chi}$, the external momentum $p$ continues to decrease while $\vec{k} \sim m_{W}$ remains constant, and the thermal modification of the Sommerfeld effect fades out. Hence the temperatures of interest are limited from below by $T_{s}$ in the range from 1 to $4 \mathrm{GeV}$. An exception is the Sommerfeld enhancement due to photon exchange between the charginos, whose effect on the relic density turns off only when the charginos decouple from the thermal plasma at a temperature set by the neutralino-chargino mass difference.

\section{A.1 Higgs vacuum expectation value}

We approximate the temperature dependence of the Higgs field vacuum expectation value by

$$
v(T)=v \sqrt{1-\frac{T^{2}}{T_{c}^{2}}} \quad\left(T<T_{c}\right)
$$

and zero above $T_{c}$. The critical temperature is taken to be $T_{c}=165 \mathrm{GeV}$, as follows from the effective potential given in ref. [68]. The expansion of the Universe proceeds adiabatically such that the particle masses are given by the standard expressions with the instantaneous value $v(T)$.

\section{A.1.1 Gauge boson masses}

This implies the temperature-dependent mass (squared) $m_{W}^{2}(T)=m_{W}^{2}+\left[\delta m_{W}^{2}\right]_{\mathrm{vev}}$ for the electroweak gauge bosons, where

$$
\left[\delta m_{W}^{2}\right]_{\mathrm{vev}}=-\frac{m_{W}^{2} T^{2}}{T_{c}^{2}}
$$

and $m_{W}^{2}(T)=0$ above $T_{c}$. Similarly for the $Z$ boson mass $m_{Z}(T)$. 

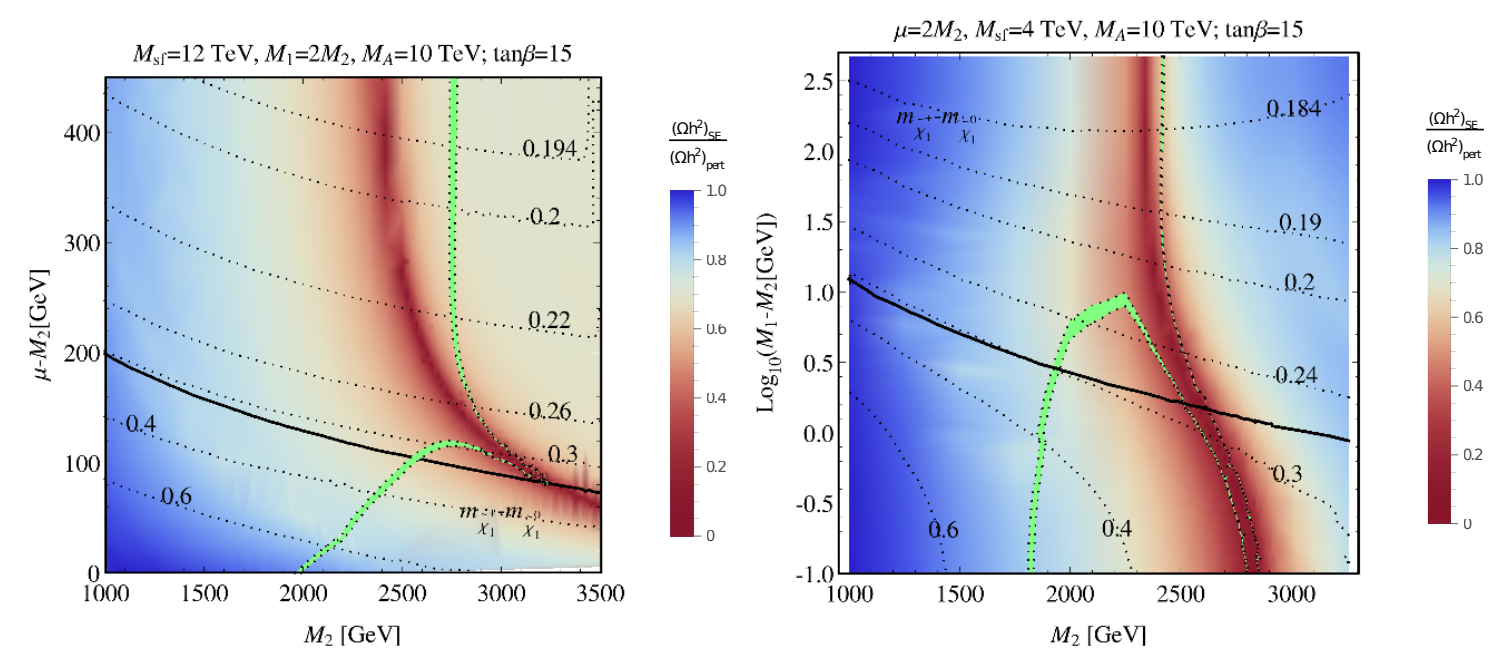

Figure 15. Contours of constant zero-temperature chargino-neutralino mass splitting (in $\mathrm{GeV}$ ) in the plane of $M_{2}$ vs. $\mu-M_{2}$ (left) and $M_{1}-M_{2}$ (right) corresponding to figures 3 and 8 . The contours include the tree-level and one-loop mass splitting. Above the thick black line the mass splitting is radiatively dominated, that is, the one-loop correction is larger than the tree-level splitting. The background refers to the size of the Sommerfeld effect and the green band to the correct relic density (within $2 \sigma$ ), as in figures 3 and 8 .

\section{A.1.2 Lightest neutralino-chargino mass difference}

The temperature dependence of the neutralino and chargino masses is not by itself of interest, since always $m_{\chi} \gg T$. However, the temperature dependence of small mass splittings must be considered, since the mass splitting determines, for instance, the location of the Sommerfeld resonance, and further appears in the nearly on-shell propagator of the two-neutralino/chargino state in the ladder diagrams.

Close to the pure-wino limit the neutralino-chargino mass difference is dominated by the radiatively induced splitting, which in the pure-wino limit is given by

$$
\left[\delta m_{+0}\right]_{\text {radiative }}=\frac{1-c_{w}}{2} \alpha_{2} m_{W} \approx 158 \mathrm{MeV} .
$$

The expression refers to the approximation $M_{Z} \ll m_{\chi}$ and the numerical value employs the $\mathrm{SU}(2)$ coupling $\alpha_{2}\left(m_{\chi}\right)=0.032810$ at the scale $m_{\chi}=2.5 \mathrm{TeV}$. Whenever the radiative mass splitting dominates over the tree-level splitting, it changes very little compared to the pure-wino value. We therefore assume that it is proportional to $v$, and implement the temperature-dependence by multiplying the zero-temperature radiative contribution to the mass difference with $v(T) / v$.

In the presence of a Higgsino- or bino- component of the wino-like neutralino, there is an additional tree-level mass splitting, which can be computed from the mass matrices $X$, $Y$ in section 2.1. The dependence on the Higgs vacuum expectation value changes from quartic towards the pure-wino limit to quadratic when the Higgsino or bino admixture increases. The cross-over to quadratic dependence occurs in about the same region in the parameter space shown in figures 3 (Higgsino admixture) and 8 (bino admixture), 
respectively, where the tree-level mass splitting becomes comparable to and then exceeds the radiative one. In case of a mixed wino-Higgsino dark matter particle, the radiative and one-loop mass splittings are equal when $\mu-M_{2}$ (assuming $\mu$ and $M_{2}$ have equal sign) is between approximately $200 \mathrm{GeV}\left(M_{2}=1 \mathrm{TeV}\right)$ and $100 \mathrm{GeV}\left(M_{2}=3.5 \mathrm{TeV}\right)$, which includes the "nose" in figure 3, where the "correct relic density" line is pulled into the Sommerfeld resonance. For mixed bino-wino dark matter, equality occurs when $M_{1}-M_{2}$ ranges from approximately $10 \mathrm{GeV}$ to $1 \mathrm{GeV}$ in the range of $M_{2}$ shown in figure 8 . This is illustrated quantitatively in figure 15 , which shows contours of constant mass splitting on the background of figures 3 and 8 .

Thus, the neutralino-chargino mass splitting has two components with different dependence on the Higgs vacuum expectation value and hence different temperature dependence. In the numerical investigation of this effect, we separate the tree and one-loop contribution to the mass splitting and modify each by its own dependence on $v(T)$.

\section{A.2 Thermal self-energies}

The second effect on the particle masses arises from their thermal self-energies. We discuss the case of electroweak gauge bosons and neutralino-chargino mass splittings in turn.

\section{A.2.1 Electroweak gauge bosons}

The potential generated by electroweak gauge boson exchange is the Fourier transform of the gauge boson propagator. Including the full gauge boson self-energy, the latter is given by

$$
\frac{1}{\vec{k}^{2}+m_{W}^{2}(T)+\Pi_{00}\left(k^{0},|\vec{k}|\right)} .
$$

The 00 component appears, since the spin-independent potential is generated by the exchange of the zero component of the gauge field. The one-loop thermal contribution to the self-energy can be represented as

$$
\left[\Pi_{00}\right]_{\text {thermal }}=\frac{g_{2}^{2}}{2 \pi^{2}} \int_{-\infty}^{\infty} d q^{0}\left|q^{0}\right| n_{X}\left(\left|q^{0}\right|\right) I_{X}\left(\frac{k^{0}}{|\vec{k}|}, \frac{q^{0}}{|\vec{k}|}, \frac{m_{W}}{|\vec{k}|}\right),
$$

where $n_{B}\left(n_{F}\right)$ denotes the Bose-Einstein (Fermi-Dirac) distribution, and $I_{X}$ arises from the loop integrand after integration over the spatial loop momentum $\vec{q} . X=B(F)$ must be chosen for gauge boson (fermion) loops.

The standard expressions for thermal gauge boson masses, which have been employed in previous investigations of thermal modifications of the Sommerfeld effect [8, 9] refer to the self-energy $\Pi_{00}$ with external momentum $k^{0},|\vec{k}| \ll T$, while in fact $|\vec{k}| \gg T$. Furthermore $\left|q^{0}\right| \sim T$, since for larger $\left|q^{0}\right|$ the integrand is exponentially suppressed by the thermal distribution function. The appropriate procedure is therefore to expand $I_{X}$ in $k^{0} /|\vec{k}|$ and $q^{0} /|\vec{k}|$.

We are not aware of computations of $\left[\Pi_{00}\right]_{\text {thermal }}$ for general external momentum in the broken electroweak gauge theory. We shall therefore estimate the self-energy in the unbroken theory, using results from ref. [69] for QCD, which is formally applicable to our 
situation when $T \gg m_{W}$, which may be realised at the beginning of freeze-out. Expanding the expressions for $I_{X}$ given in section III of ref. [69], we find

$$
\left[\Pi_{00}\right]_{\text {thermal }}=-\frac{g_{2}^{2} T^{2}}{9},
$$

up to corrections of order $T^{2} /|\vec{k}|^{2} \sim T / m_{\chi}$. This result arises from gauge boson (and ghost) loops only. The fermion loop contribution is suppressed. In the broken theory with massive gauge bosons in the loop, the expression above will be multiplied by some function $f\left(m_{W}(T) /|\vec{k}|\right)$ with $f(0)=1$. It is reasonable to assume that a massive propagator will suppress the loop integral such that $0<f(x) \leq 1$. The above estimate therefore provides an upper limit on the thermal self-energy modification of the propagator (A.4).

The self-energy correction (A.6) is opposite in sign and has a smaller coefficient than the usual thermal mass, making it less relevant. With regard to eq. (A.4) we note that it has the same temperature dependence and sign as the Higgs vacuum expectation value effect (A.2), but the coefficient of eq. (A.6) is about five times smaller as long as $T<T_{c}$. Above $T_{c},\left[\delta m_{W}^{2}\right]_{\mathrm{vev}}=-m_{W}^{2}$ remains constant and is eventually exceeded by the thermal self-energy contribution. This is not relevant for the Sommerfeld effect in the dark matter mass range considered here, so we may assume that the dominant effect on the electroweak Yukawa potential is due to eq. (A.2). ${ }^{11}$

\section{A.2.2 Neutralino-chargino mass difference}

The thermal one-loop self-energy of a fermion in a vector-like theory with massless gauge bosons was considered in ref. [70]. The extension to massive gauge boson exchange and the full electroweak theory can be found in refs. [71] and [72], respectively. The latter reference also covers the MSSM, which features loop diagrams with gauge boson and Higgs exchange, as well as a fermion-sfermion loop.

The thermal correction to the fermion mass is obtained by expanding the self-energy in $\vec{k} / m_{\chi}$, where $k$ is the external momentum, and by solving the dispersion relation for $\omega(\vec{k}=$ $0)=m_{\chi}+\left[\delta m_{\chi}\right]_{\text {thermal }}$. For the photon radiative correction to the chargino mass we find

$$
\left[\delta m_{\chi^{+}}\right]_{\mathrm{thermal}, \gamma}=\frac{\pi \alpha_{\mathrm{em}}}{3} \frac{T^{2}}{m_{\chi}}
$$

and zero for the neutralino. This generates a mass difference, which is of order $50 \mathrm{MeV}$ at the beginning of freeze-out, smaller than but in a similar ballpark as the zero-temperature radiative mass splitting. However, the thermal correction decreases rapidly with $T$.

Because of this, we do not evaluate the mass splitting in the full MSSM. Rather, we give some estimates. First, to obtain an idea of the effect of a non-zero gauge boson mass

\footnotetext{
${ }^{11} \mathrm{As}$ an aside we note that in the unbroken gauge theory with massless gauge bosons, the negative value of eq. (A.6) seems to lead to a singularity in $1 /\left(\vec{k}^{2}+\Pi_{00}\right)$. However, eq. (A.6) has been derived under the assumption $T \ll|\vec{k}|$, implying $\left|\Pi_{00}\right| \ll \vec{k}^{2}$, hence the singularity arises for values of $\vec{k}$ outside the approximations made. The thermal correction to the propagator is equivalent to $-\left[\Pi_{00}\right]_{\text {thermal }} / \vec{k}^{4}$ up to corrections beyond the adopted approximation, which in coordinate space amounts to a correction of the form $\alpha_{2}^{2} T^{2} r$ on top of the $\alpha_{2} / r$ Coulomb potential.
} 
we generalise the above expression to the case of a massive photon using results from refs. $[71,72]$. We find that the right-hand side of eq. (A.7) is multiplied by the factor

$$
r\left(x_{T}\right)=\frac{6}{\pi^{2}} \int_{x_{T}}^{\infty} d x \frac{\sqrt{x^{2}-x_{T}^{2}}}{e^{x}-1}\left(1+\frac{x_{T}^{2}}{2 x^{2}}\right),
$$

where $x_{T}=m_{\gamma} / T$. The second term never exceeds a few percent of the first. The modifying factor equals 1 at $x_{T}=0$ by definition, reaches $r(1) \approx 0.4$ and is exponentially suppressed for $x_{T}>1$ as expected. The electroweak gauge boson contribution to the chargino and neutralino self-energy is suppressed by $r\left(x_{T}\right)$ relative to photon exchange, but is multiplied by the larger $\mathrm{SU}(2)$ coupling. Hence it can compete with (A.7) at the beginning of freezeout if the dark matter mass is above $2.5 \mathrm{TeV}$, but is exponentially suppressed very soon after. Similar conclusions apply to Higgs exchange.

Finally, we consider the fermion-sfermion loop. The relevant case is the thermal correction to the fermion propagator, which can be assumed to be massless, since the top quark is too heavy to be relevant. We find

$$
\left[\delta m_{\chi}\right]_{\text {thermal, }(\mathrm{s}) \text { fermion }}=\text { coupling factors } \times \text { const } \times \frac{m_{\chi} T^{4}}{\left(M_{\mathrm{sf}}^{2}-m_{\chi}^{2}\right)^{2}} .
$$

We recall that to avoid sfermion co-annihilation, we assumed $M_{\mathrm{sf}}>1.25 m_{\chi}$ in our analysis. The last factor is therefore parametrically of order $T^{4} / m_{\chi}^{3}$, which makes this contribution negligible compared to the photon correction. We may therefore assume that the photon correction (A.7) is the only relevant contribution to the neutralino-chargino mass splitting, except perhaps at the beginning of freeze-out, where it still provides a reasonable estimate up to $\mathcal{O}(1)$ factors.

\section{A.3 Effect on the Sommerfeld enhancement and relic density}

We proceed to estimating the thermal modification of the Sommerfeld effect and its consequences for the relic density. For wino-like dark matter the Sommerfeld effect arises primarily from ladder diagram topologies with the exchange of $W$ bosons. The loop momentum is in the potential region satisfying $k^{0} \ll|\vec{k}| \ll m_{\chi}$. With $\vec{p} \ll m_{\chi}$ the external momentum of the ladder diagram, we can estimate the magnitude of the contribution of each ladder rung from the loop momentum region $|\vec{k}| \sim \lambda|\vec{p}|$ as

$$
I \sim \frac{\pi \alpha_{2} m_{\chi}}{|\vec{p}|} \times \frac{\lambda^{3}}{\left(\lambda^{2}+\frac{m_{W}^{2}(T)}{|\vec{p}|^{2}}\right)\left(\lambda^{2}+\lambda+\frac{m_{\chi} \delta m_{+}(T)}{|\vec{p}|^{2}}\right)},
$$

which multiplies the tree annihilation cross section. The Sommerfeld enhancement is nonperturbative and large when $I$ becomes of order 1.

Several well-known results follow immediately from this equation. (1) When $m_{W}=0$, $\delta m_{+0}=0$, the largest contribution arises from $|\vec{k}| \sim|\vec{p}| \sim m_{\chi} v$ (that is, $\lambda \sim 1$ ), and the Coulomb enhancement $\pi \alpha_{2} / v$ is recovered. (2) When $m_{W} \neq 0$, but still $\delta m_{+0}=0$, the same holds as long as $|\vec{p}| \gg m_{W}$. As soon as $|\vec{p}| \sim m_{W}$ or smaller, the largest contribution is obtained from $\lambda \sim m_{W} /|\vec{p}|$, and $I \sim \pi \alpha_{2} m_{\chi} / m_{W}$ independent of the external momentum. 
This is the saturation regime for the Sommerfeld enhancement of the Yukawa potential. (3) The neutralino-chargino mass difference provides an $\mathcal{O}(1)$ modification of the Sommerfeld enhancement factor $I$ whenever $m_{\chi} \delta m_{+0} \sim \max \left(|\vec{p}|^{2}, m_{W}^{2}\right)$ and reduces or cuts off the enhancement when $m_{\chi} \delta m_{+0}$ is larger than the right-hand side of this relation.

In the following we use the above expression to estimate the impact of the thermal modifications of $m_{W}(T)$ and $\delta m_{+0}(T)$ discussed in the previous subsection. In doing so, we correlate the external neutralino or chargino momentum with the temperature of the Universe according to $\vec{p}^{2} \sim m_{\chi} T=m_{\chi}^{2} / x$. We further support these estimates by implementing the thermal effects into our Sommerfeld code as described below.

\section{A.3.1 Yukawa potential}

We first study the modification of the Yukawa potential generated by $W$ exchange. As discussed above, the leading effect is the temperature dependence of the Higgs vacuum expectation value. The thermal self-energy correction has the same temperature dependence, but is smaller. The modification of $I$ is due to the $W$ propagator

$$
\frac{1}{\max \left(m_{\chi} T, m_{W}^{2}\right)+m_{W}^{2}-T^{2} / 4},
$$

where we assume $T<T_{c}$ and approximate $m_{W}^{2} / T_{c}^{2} \rightarrow 1 / 4$. At the beginning of freeze-out when $m_{\chi} T>m_{W}^{2}$, the relative size of the thermal correction is $1 /(4 x)$ which for $x_{f} \sim 20$ does not exceed $1.2 \%$. When saturation is reached at $T_{s} \sim m_{W}^{2} / m_{\chi}$, the relative correction is only of order $m_{W}^{2} /\left(4 m_{\chi}^{2}\right) \sim 0.02 \%$ for a reference dark matter mass $m_{\chi}=2.5 \mathrm{TeV}$.

An analytic expression for the Sommerfeld effect is available in a one-state model, when the Yukawa potential is replaced by the so-called Hulthén potential, which provides a good approximation [73]. Using this expression we find a maximal change of the Sommerfeld factor of $0.2 \%$ at the beginning of freeze-out and decreasing afterwards, confirming the above simple estimate.

The modification of the relic density is expected to be even smaller, since the suppression due to the Sommerfeld effect builds up from the beginning of freeze-out, where it is least significant, until about $x \sim 10^{4}$, where annihilations terminate, see for instance figure 4 of ref. [15]. We have implemented the thermal modification of the potential in our code to check this explicitly. The modification is CPU expensive, since the Sommerfeldcorrected cross section, which is thermally averaged for given $T$, must now be recomputed for every value of $T$. We created two-dimensional cross section tables in velocity and temperature, adopting 59 temperature points. We then compute the Sommerfeld effect and relic density including the temperature-dependent potentials for the Higgsino-to-wino trajectory in MSSM parameter space considered in ref. [15], to which we refer for details on these models. For the mostly wino models 8 to 13 of the trajectory, we find that the relic density change is below one permille in all cases, in good agreement with the above estimates, and not visible within the numerical accuracy of the code.

\section{A.3.2 Neutralino-chargino mass splitting}

It is evident from the temperature dependence of the two contributions (vacuum expectation value and thermal self-energy) that the largest relative effect of the thermal correction 
to the neutralino-chargino mass splitting again arises at the beginning of freeze-out. This has two immediate consequences. (1) While the Sommerfeld resonance depends sensitively on the mass splitting (see, for instance the two-state model in ref. [74], which shares the essential features regarding the mass splitting with wino-like MSSM models), the resonance effect develops sufficiently late after the beginning of freeze-out. We therefore conclude that the thermal effect on the resonance region is negligible. (2) At the beginning of freeze-out $\lambda \sim 1$ in (A.10), hence the relative modification of $I$ due to the mass splitting is of order

$$
\frac{m_{\chi} \delta m_{+0}(T)}{|\vec{p}|^{2}} \sim \frac{\left[\delta m_{+0}\right]_{\mathrm{vev}+\text { thermal }}}{T} \approx \text { few permille }
$$

as the thermal correction to the mass splitting is as large as the mass splitting itself. The numerical estimate is based on the assumption that the entire zero-temperature mass difference of up to $0.5 \mathrm{GeV}$ vanishes due to the vanishing of the Higgs expectation value at $T=T_{c}$, which gives the largest possible effect.

We studied the impact of the temperature-dependent neutralino-chargino mass splitting on the relic density with the extended numerical code described above for the wino-like trajectory models of ref. [15]. The thermal modification is again in the permille range, in agreement with the analytic estimates, reaching $0.7 \%$ at maximum. Once again we find that the observed thermal effect is of the same order at the numerical uncertainties due to sampling and the choice of $x_{\infty}$, hence we can only state that the thermal effect is well below $1 \%$ in all cases studied.

\section{A.3.3 Summary}

We conclude that thermal modifications of the Sommerfeld effect change the relic density at most in the upper permille range, which is negligible for all practical purposes. We point out that our investigation of thermal effects is not complete. For example, we did not discuss the direct modification of the neutralino and chargino two-particle wave function due to interactions with gauge bosons in the thermal plasma, an effect that would be referred to as "dissociation" by soft gauge bosons in the case of bound states. Power counting suggests that this effect is of the same order as the ones investigated here. However, since all these are far smaller than the theoretical uncertainty from perturbative higher-order corrections, which is probably a few percent, we do not attempt a complete analysis in this work.

Open Access. This article is distributed under the terms of the Creative Commons Attribution License (CC-BY 4.0), which permits any use, distribution and reproduction in any medium, provided the original author(s) and source are credited.

\section{References}

[1] M. Cirelli, N. Fornengo and A. Strumia, Minimal dark matter, Nucl. Phys. B 753 (2006) 178 [hep-ph/0512090] [INSPIRE].

[2] A. Fowlie, K. Kowalska, L. Roszkowski, E.M. Sessolo and Y.-L.S. Tsai, Dark matter and collider signatures of the MSSM, Phys. Rev. D 88 (2013) 055012 [arXiv:1306.1567] [INSPIRE]. 
[3] M. Cahill-Rowley et al., Complementarity of dark matter searches in the phenomenological MSSM, Phys. Rev. D 91 (2015) 055011 [arXiv:1405.6716] [INSPIRE].

[4] E.A. Bagnaschi et al., Supersymmetric dark matter after LHC run 1, Eur. Phys. J. C 75 (2015) 500 [arXiv: 1508.01173] [INSPIRE].

[5] K.J. de Vries et al., The pMSSM10 after LHC run 1, Eur. Phys. J. C 75 (2015) 422 [arXiv: 1504.03260] [INSPIRE].

[6] J. Hisano, S. Matsumoto, M.M. Nojiri and O. Saito, Non-perturbative effect on dark matter annihilation and gamma ray signature from galactic center, Phys. Rev. D 71 (2005) 063528 [hep-ph/0412403] [INSPIRE].

[7] J. Hisano, S. Matsumoto, M. Nagai, O. Saito and M. Senami, Non-perturbative effect on thermal relic abundance of dark matter, Phys. Lett. B 646 (2007) 34 [hep-ph/0610249] [INSPIRE].

[8] M. Cirelli, A. Strumia and M. Tamburini, Cosmology and astrophysics of minimal dark matter, Nucl. Phys. B 787 (2007) 152 [arXiv:0706.4071] [INSPIRE].

[9] A. Hryczuk, R. Iengo and P. Ullio, Relic densities including Sommerfeld enhancements in the MSSM, JHEP 03 (2011) 069 [arXiv:1010.2172] [INSPIRE].

[10] A. Hryczuk and R. Iengo, The one-loop and Sommerfeld electroweak corrections to the wino dark matter annihilation, JHEP 01 (2012) 163 [Erratum ibid. 06 (2012) 137] [arXiv:1111.2916] [INSPIRE].

[11] A. Hryczuk, The Sommerfeld enhancement for scalar particles and application to sfermion co-annihilation regions, Phys. Lett. B 699 (2011) 271 [arXiv:1102.4295] [INSPIRE].

[12] M. Beneke, C. Hellmann and P. Ruiz-Femenía, Non-relativistic pair annihilation of nearly mass degenerate neutralinos and charginos I. General framework and S-wave annihilation, JHEP 03 (2013) 148 [Erratum ibid. 10 (2013) 224] [arXiv:1210.7928] [INSPIRE].

[13] C. Hellmann and P. Ruiz-Femenía, Non-relativistic pair annihilation of nearly mass degenerate neutralinos and charginos II. P-wave and next-to-next-to-leading order S-wave coefficients, JHEP 08 (2013) 084 [arXiv:1303.0200] [INSPIRE].

[14] M. Beneke, C. Hellmann and P. Ruiz-Femenía, Non-relativistic pair annihilation of nearly mass degenerate neutralinos and charginos III. Computation of the Sommerfeld enhancements, JHEP 05 (2015) 115 [arXiv:1411.6924] [INSPIRE].

[15] M. Beneke, C. Hellmann and P. Ruiz-Femenía, Heavy neutralino relic abundance with Sommerfeld enhancements - a study of pMSSM scenarios, JHEP 03 (2015) 162 [arXiv: 1411.6930] [INSPIRE].

[16] A. Hryczuk, I. Cholis, R. Iengo, M. Tavakoli and P. Ullio, Indirect detection analysis: wino dark matter case study, JCAP 07 (2014) 031 [arXiv:1401.6212] [INSPIRE].

[17] T. Cohen, M. Lisanti, A. Pierce and T.R. Slatyer, Wino dark matter under siege, JCAP 10 (2013) 061 [arXiv:1307.4082] [INSPIRE].

[18] J. Fan and M. Reece, In wino veritas? Indirect searches shed light on neutralino dark matter, JHEP 10 (2013) 124 [arXiv: 1307.4400] [INSPIRE].

[19] G. Bélanger, C. Boehm, M. Cirelli, J. Da Silva and A. Pukhov, PAMELA and FERMI-LAT limits on the neutralino-chargino mass degeneracy, JCAP 11 (2012) 028 [arXiv:1208.5009] [INSPIRE].

[20] B. Bhattacherjee, M. Ibe, K. Ichikawa, S. Matsumoto and K. Nishiyama, Wino dark matter and future dSph observations, JHEP 07 (2014) 080 [arXiv: 1405.4914] [INSPIRE]. 
[21] E.J. Chun and J.-C. Park, Electro-weak dark matter: non-perturbative effect confronting indirect detections, Phys. Lett. B 750 (2015) 372 [arXiv:1506.07522] [INSPIRE].

[22] K. Harigaya, K. Kaneta and S. Matsumoto, Gaugino coannihilations, Phys. Rev. D 89 (2014) 115021 [arXiv:1403.0715] [INSPIRE].

[23] S. Heinemeyer, W. Hollik and G. Weiglein, FeynHiggs: a program for the calculation of the masses of the neutral CP even Higgs bosons in the MSSM, Comput. Phys. Commun. 124 (2000) 76 [hep-ph/9812320] [INSPIRE].

[24] G. Degrassi, S. Heinemeyer, W. Hollik, P. Slavich and G. Weiglein, Towards high precision predictions for the MSSM Higgs sector, Eur. Phys. J. C 28 (2003) 133 [hep-ph/0212020] [INSPIRE].

[25] G. Bélanger et al., Indirect search for dark matter with MicrOMEGAs2.4, Comput. Phys. Commun. 182 (2011) 842 [arXiv: 1004.1092] [INSPIRE].

[26] G. Bélanger, F. Boudjema, A. Pukhov and A. Semenov, MicrOMEGAs 3 : a program for calculating dark matter observables, Comput. Phys. Commun. 185 (2014) 960 [arXiv: 1305.0237] [INSPIRE].

[27] ATLAS and CMS collaborations, Combined measurement of the Higgs boson mass in pp collisions at $\sqrt{s}=7$ and $8 \mathrm{TeV}$ with the ATLAS and CMS experiments, Phys. Rev. Lett. 114 (2015) 191803 [arXiv: 1503.07589] [INSPIRE].

[28] T. Hahn, S. Heinemeyer, W. Hollik, H. Rzehak and G. Weiglein, High-precision predictions for the light CP-even Higgs boson mass of the minimal supersymmetric standard model, Phys. Rev. Lett. 112 (2014) 141801 [arXiv:1312.4937] [INSPIRE].

[29] M. Carena, J.R. Espinosa, M. Quirós and C.E.M. Wagner, Analytical expressions for radiatively corrected Higgs masses and couplings in the MSSM, Phys. Lett. B 355 (1995) 209 [hep-ph/9504316] [INSPIRE].

[30] H.E. Haber, R. Hempfling and A.H. Hoang, Approximating the radiatively corrected Higgs mass in the minimal supersymmetric model, Z. Phys. C 75 (1997) 539 [hep-ph/9609331] [INSPIRE].

[31] S. Heinemeyer, W. Hollik and G. Weiglein, Electroweak precision observables in the minimal supersymmetric standard model, Phys. Rept. 425 (2006) 265 [hep-ph/0412214] [INSPIRE].

[32] Particle Data Group collaboration, K.A. Olive et al., Review of particle physics, Chin. Phys. C 38 (2014) 090001 [InSPIRE].

[33] S. Stone, New physics from flavour, PoS (ICHEP2012) 033 [arXiv:1212.6374] [INSPIRE].

[34] M. Misiak and M. Steinhauser, NNLO QCD corrections to the $\bar{B} \rightarrow X_{s} \gamma$ matrix elements using interpolation in $m_{c}$, Nucl. Phys. B 764 (2007) 62 [hep-ph/0609241] [INSPIRE].

[35] LHCb and CMS collaborations, Observation of the rare $B_{s}^{0} \rightarrow \mu^{+} \mu^{-}$decay from the combined analysis of CMS and LHCb data, Nature $\mathbf{5 2 2}$ (2015) 68 [arXiv:1411.4413] [INSPIRE].

[36] A.J. Buras, J. Girrbach, D. Guadagnoli and G. Isidori, On the standard model prediction for $B R\left(B_{s, d} \rightarrow \mu^{+} \mu^{-}\right)$, Eur. Phys. J. C 72 (2012) 2172 [arXiv:1208.0934] [InSPIRE].

[37] BABAR collaboration, J.P. Lees et al., Evidence of $B^{+} \rightarrow \tau^{+} \nu$ decays with hadronic $B$ tags, Phys. Rev. D 88 (2013) 031102 [arXiv: 1207.0698] [INSPIRE].

[38] BELLE collaboration, I. Adachi et al., Evidence for $B^{-} \rightarrow \tau^{-} \bar{\nu}_{\tau}$ with a hadronic tagging method using the full data sample of Belle, Phys. Rev. Lett. 110 (2013) 131801 [arXiv: 1208.4678] [INSPIRE]. 
[39] W. Altmannshofer, M. Carena, N.R. Shah and F. Yu, Indirect probes of the MSSM after the Higgs discovery, JHEP 01 (2013) 160 [arXiv:1211.1976] [INSPIRE].

[40] J.M. Frere, D.R.T. Jones and S. Raby, Fermion masses and induction of the weak scale by supergravity, Nucl. Phys. B 222 (1983) 11 [inSPIRE].

[41] M. Claudson, L.J. Hall and I. Hinchliffe, Low-energy supergravity: false vacua and vacuous predictions, Nucl. Phys. B 228 (1983) 501 [InSPIRE].

[42] M. Drees and M.M. Nojiri, The neutralino relic density in minimal $N=1$ supergravity, Phys. Rev. D 47 (1993) 376 [hep-ph/9207234] [InSPIRE].

[43] P. Nath and R.L. Arnowitt, Predictions in SU(5) supergravity grand unification with proton stability and relic density constraints, Phys. Rev. Lett. 70 (1993) 3696 [hep-ph/9302318] [INSPIRE].

[44] LUX collaboration, D.S. Akerib et al., First results from the LUX dark matter experiment at the Sanford Underground Research Facility, Phys. Rev. Lett. 112 (2014) 091303 [arXiv:1310.8214] [INSPIRE].

[45] T. Fritzsche and W. Hollik, Complete one loop corrections to the mass spectrum of charginos and neutralinos in the MSSM, Eur. Phys. J. C 24 (2002) 619 [hep-ph/0203159] [InSPIRE].

[46] A.C. Fowler and G. Weiglein, Precise predictions for Higgs production in neutralino decays in the complex MSSM, JHEP 01 (2010) 108 [arXiv:0909.5165] [INSPIRE].

[47] A.C. Fowler, Higher order and CP-violating effects in the neutralino and Higgs boson sectors of the MSSM, Ph.D. thesis, Durham University, Durham U.K. (2010) [InSPIRE].

[48] A. Bharucha, A. Fowler, G. Moortgat-Pick and G. Weiglein, Consistent on shell renormalisation of electroweakinos in the complex MSSM: LHC and LC predictions, JHEP 05 (2013) 053 [arXiv: 1211.3134] [INSPIRE].

[49] A. Bharucha, S. Heinemeyer, F. von der Pahlen and C. Schappacher, Neutralino decays in the complex MSSM at one-loop: a comparison of on-shell renormalization schemes, Phys. Rev. D 86 (2012) 075023 [arXiv:1208.4106] [INSPIRE].

[50] T. Hahn, Generating Feynman diagrams and amplitudes with FeynArts 3, Comput. Phys. Commun. 140 (2001) 418 [hep-ph/0012260] [INSPIRE].

[51] T. Hahn and C. Schappacher, The implementation of the minimal supersymmetric standard model in FeynArts and FormCalc, Comput. Phys. Commun. 143 (2002) 54 [hep-ph/0105349] [INSPIRE].

[52] T. Hahn and M. Pérez-Victoria, Automatized one loop calculations in four-dimensions and D-dimensions, Comput. Phys. Commun. 118 (1999) 153 [hep-ph/9807565] [INSPIRE].

[53] T. Fritzsche, T. Hahn, S. Heinemeyer, F. von der Pahlen, H. Rzehak and C. Schappacher, The implementation of the renormalized complex MSSM in FeynArts and FormCalc, Comput. Phys. Commun. 185 (2014) 1529 [arXiv:1309.1692] [INSPIRE].

[54] A. Chatterjee, M. Drees, S. Kulkarni and Q. Xu, On the on-shell renormalization of the chargino and neutralino masses in the MSSM, Phys. Rev. D 85 (2012) 075013 [arXiv: 1107.5218] [INSPIRE].

[55] C. Hellmann, Sommerfeld corrections in neutralino dark matter pair-annihilations and relic abundance in the general MSSM, Ph.D. thesis, Technische Universität München, Munich Germany (2015). 
[56] P. Gondolo and G. Gelmini, Cosmic abundances of stable particles: improved analysis, Nucl. Phys. B 360 (1991) 145 [InSPIRE].

[57] P. Gondolo, J. Edsjö, P. Ullio, L. Bergstrom, M. Schelke and E.A. Baltz, DarkSUSY: computing supersymmetric dark matter properties numerically, JCAP 07 (2004) 008 [astro-ph/0406204] [INSPIRE].

[58] Particle Data Group collaboration, J. Beringer et al., Review of particle physics (RPP), Phys. Rev. D 86 (2012) 010001 [inSPIRE].

[59] H. Baer, A. Mustafayev, E.-K. Park and S. Profumo, Mixed wino dark matter: consequences for direct, indirect and collider detection, JHEP 07 (2005) 046 [hep-ph/0505227] [INSPIRE].

[60] Planck collaboration, P.A.R. Ade et al., Planck 2015 results. XIII. Cosmological parameters, arXiv:1502.01589 [INSPIRE].

[61] J. Hisano, S. Matsumoto and M.M. Nojiri, Explosive dark matter annihilation, Phys. Rev. Lett. 92 (2004) 031303 [hep-ph/0307216] [INSPIRE].

[62] J. Hisano, S. Matsumoto, O. Saito and M. Senami, Heavy wino-like neutralino dark matter annihilation into antiparticles, Phys. Rev. D 73 (2006) 055004 [hep-ph/0511118] [INSPIRE].

[63] L. Roszkowski, E.M. Sessolo and A.J. Williams, Prospects for dark matter searches in the pMSSM, JHEP 02 (2015) 014 [arXiv:1411.5214] [INSPIRE].

[64] M.E. Cabrera-Catalan, S. Ando, C. Weniger and F. Zandanel, Indirect and direct detection prospect for TeV dark matter in the nine parameter MSSM, Phys. Rev. D 92 (2015) 035018 [arXiv: 1503.00599] [INSPIRE].

[65] J. Bramante, N. Desai, P. Fox, A. Martin, B. Ostdiek and T. Plehn, Towards the final word on neutralino dark matter, arXiv: 1510.03460 [INSPIRE].

[66] M. Beneke, A. Bharucha, F. Dighera, A. Hryczuk, S. Recksiegel and P. Ruiz-Femenía, work in progress.

[67] M. Beneke, F. Dighera and A. Hryczuk, Relic density computations at NLO: infrared finiteness and thermal correction, JHEP 10 (2014) 045 [arXiv:1409.3049] [INSPIRE].

[68] M. Quirós, Finite temperature field theory and phase transitions, in High energy physics and cosmology. Proceedings, Summer School, Trieste Italy June 29-July 17 1998, pg. 187 [hep-ph/9901312] [INSPIRE].

[69] N. Brambilla, J. Ghiglieri, A. Vairo and P. Petreczky, Static quark-antiquark pairs at finite temperature, Phys. Rev. D 78 (2008) 014017 [arXiv:0804.0993] [INSPIRE].

[70] H.A. Weldon, Effective fermion masses of order gT in high temperature gauge theories with exact chiral invariance, Phys. Rev. D 26 (1982) 2789 [InSPIRE].

[71] E. Petitgirard, Massive fermion dispersion relation at finite temperature, Z. Phys. C 54 (1992) 673 [INSPIRE].

[72] C. Quimbay and S. Vargas-Castrillon, Fermionic dispersion relations in the standard model at finite temperature, Nucl. Phys. B 451 (1995) 265 [hep-ph/9504410] [INSPIRE].

[73] S. Cassel, Sommerfeld factor for arbitrary partial wave processes, J. Phys. G 37 (2010) 105009 [arXiv: 0903.5307] [INSPIRE].

[74] T.R. Slatyer, The Sommerfeld enhancement for dark matter with an excited state, JCAP 02 (2010) 028 [arXiv:0910.5713] [INSPIRE]. 